\title{
Ethics of Al-Enabled Recruiting and Selection: A Review and Research Agenda
}

\author{
Anna Lena Hunkenschroer ${ }^{1}$ (I) $\cdot$ Christoph Luetge $^{1}$
}

Received: 24 September 2020 / Accepted: 25 January 2022 / Published online: 8 February 2022

(c) The Author(s) 2022

\begin{abstract}
Companies increasingly deploy artificial intelligence (AI) technologies in their personnel recruiting and selection process to streamline it, making it faster and more efficient. AI applications can be found in various stages of recruiting, such as writing job ads, screening of applicant resumes, and analyzing video interviews via face recognition software. As these new technologies significantly impact people's lives and careers but often trigger ethical concerns, the ethicality of these AI applications needs to be comprehensively understood. However, given the novelty of AI applications in recruiting practice, the subject is still an emerging topic in academic literature. To inform and strengthen the foundation for future research, this paper systematically reviews the extant literature on the ethicality of AI-enabled recruiting to date. We identify 51 articles dealing with the topic, which we synthesize by mapping the ethical opportunities, risks, and ambiguities, as well as the proposed ways to mitigate ethical risks in practice. Based on this review, we identify gaps in the extant literature and point out moral questions that call for deeper exploration in future research.
\end{abstract}

Keywords Artificial intelligence $\cdot$ Algorithmic hiring $\cdot$ Employee selection $\cdot$ Ethical recruitment $\cdot$ Ethics of AI $\cdot$ Bias of AI

\section{Introduction}

Pursuant to advances in technological developments, artificial intelligence (AI) has expanded into various business sectors and workplaces. Along with such fields as the prediction of credit worthiness, criminal justice systems, and pricing of goods, AI-enabled technologies have disrupted companies' personnel recruiting and selection practices, entering the market at exponential rates (Yarger et al., 2020). AI-advanced selection tools are attractive for organizations, due to their higher speed and efficiency gains compared with traditional screening and assessment practices (van Esch \& Black, 2019) and are considered a valuable asset in today's "war for talent" (Leicht-Deobald et al., 2019, p. 381). Today's trend toward more remote and home office work further spurs the adoption of alternatives to in-person job interviews to assess candidates remotely (Wiggers, 2020).

Anna Lena Hunkenschroer

Anna.Hunkenschroer@tum.de

Christoph Luetge

Luetge@tum.de

1 Chair of Business Ethics, Technical University of Munich, Arcisstr. 21, 80333 Munich, Germany
We define AI recruiting as any procedure that makes use of AI for the purposes of assisting organizations during the recruitment and selection of job candidates, whereas $A I$ can be defined as "a system's ability to interpret external data correctly, to learn from such data, and to use those learnings to achieve specific goals and tasks through flexible adaptation" (Kaplan \& Haenlein, 2019, p. 17). We thereby refer to a broad concept of AI that includes complex machine learning approaches such as deep neural networks, but also covers simple algorithms relying on regression analyses as well as other kinds of algorithms, such as natural language processing or voice recognition.

As the outcomes of hiring decisions have serious consequences for individuals, informing where they live and how much they earn (Raghavan et al., 2020), AI recruiting practices are worth considering from an ethical perspective. Vendors of AI recruiting systems advertise that their software makes hiring decisions not only more efficient and accurate, but also fairer and less biased, as they are free of human intuition. However, AI applications in the recruiting context may generate serious conflicts with what society typically considers ethical (Tambe et al., 2019). This was illustrated by the case of Amazon in 2018, when the company abandoned its tested hiring algorithm, which had turned out to be biased and discriminatory against women 
(e.g., Mujtaba \& Mahapatra, 2019). When people are "users" or "consumers" of algorithmic systems (e.g., when following a recommendation of their movie streaming service), issues with the accuracy of recommendations against their taste may be no more than an inconvenience. However, when AI decisions are incorporated into hiring processes, applicants cannot reject them, and inaccuracies become problematic (Lee, 2018).

Although research on AI recruiting has increased substantially in recent years, a comprehensive ethical understanding of recruiting as an expanding application context of $\mathrm{AI}$ is still lacking. While the subject of algorithmic bias in hiring decisions has attracted broad interest among researchers, especially from a legal and technical perspective, there are more ethical concerns related to AI recruiting, such as data privacy, transparency, and accountability, which are worth discussing. To establish a common foundation for future research in the field, it is crucial to synthesize extant theoretical and empirical approaches to assess the ethicality of AI-powered recruiting. We address this need in our paper.

Through a systematic review of extant literature, we take a first step to offer an overview of the various ethical considerations in AI-enabled recruiting and selection. The inherent multidisciplinary nature of AI recruiting has led to a broad view of the phenomenon. Thus, we categorize extant research that considers the ethicality of AI recruiting from theoretical, practitioner, legal, technical, and descriptive perspectives. Furthermore, we provide an overview of the different AI applications along the recruiting stages, show where major ethical opportunities and risks arise and outline the proposed ways of mitigating such risks in practice. Due to the huge impact of recruiting decisions on people's lives, it is crucial that companies understand both the opportunities and the potential risks that AI recruiting technologies may create and how algorithmic decisions may be in complete disagreement with what they want to achieve.

We observe that the field of AI recruiting suffers from four shortcomings: First, there are only a few papers that provide a theoretical foundation for the ethical discussion, leaving many arguments unfounded. Additional theoretical, normative work in this area could prove beneficial to managers and organizations by providing guidance beyond mere casuistry in determining the right course of action. Second, most papers focus on the challenge of algorithmic bias, neglecting other ethical concerns, such as accountability and human autonomy in the AI recruiting context. Third, the established approaches to mitigate ethical risks are rather general and lack concrete domain-specific implementation guidelines for the recruiting context. However, a domain-specific focus is desirable, as general normative guidelines often lack tangible impact due to their superficiality (Hagendorff, 2020). Finally, we identified only limited empirical research on the topic, as well as inconsistent findings on people's fairness perceptions of AI recruiting, revealing room for further empirical research in the field to prove theoretical assumptions and derive implications for practice.

Our paper contributes to the literature in three ways. First, we comprehensively organize the extant research on ethical considerations of AI recruiting by identifying and summarizing the different perspectives taken. Second, we make accessible to researchers and human resources (HR) professionals an overview of the ethical considerations in AI recruiting by synthesizing extant research. We thereby ethically evaluate these considerations and classify them into ethical opportunities, risks, and ambiguities, developing an ethical framework of AI recruiting. Third, we identify current research gaps and propose moral topics and questions that call for a deeper exploration in both theoretical and empirical future research.

The remainder of our paper is organized as follows. First, we specify our research method and selection criteria. Afterwards, we systematically review the literature on the ethicality of AI recruiting, organizing the identified literature according to underlying perspectives. We then outline the different AI applications in the recruiting process and map ethical considerations in the form of ethical opportunities, risks, and ambiguities. In the "Discussion" Section, we summarize and discuss our review findings, by outlining considerations for future theoretical and empirical research and highlighting implications for practice. Finally, we provide concluding thoughts.

\section{Research Method}

In our review, we employ an interpretative approach according to Noblit and Hare (1988), like other literature reviews with aims similar to ours (e.g., Seele et al., 2019; Suddaby et al., 2017). In contrast to integrative reviews, which are most appropriate to summarize quantitative studies with data and construct similarity, interpretative reviews are thematic and applicable to a diverse body of literature, constituting of qualitative, quantitative, and conceptual works (Noblit \& Hare, 1988). We systematically review the literature on the ethicality of AIenabled recruiting and selection practices in four stages: First, to show how the ethicality of AI recruiting is assessed in extant research, we categorize the identified literature according to the perspectives assumed. Second, to afford a profound understanding of the underlying research topic, we give an overview of AI applications in recruiting. Third, we map the ethical considerations found in extant literature in the form of ethical opportunities, ethical risks, and ethical ambiguities. Fourth, we outline the mentioned approaches to mitigate ethical risks in practice. Figure 1 outlines the research design of this review paper.

\section{Criteria for Selection, Inclusion, and Exclusion}

On January 4, 2021, we performed a structured keywordbased literature search in the major online databases: Business 


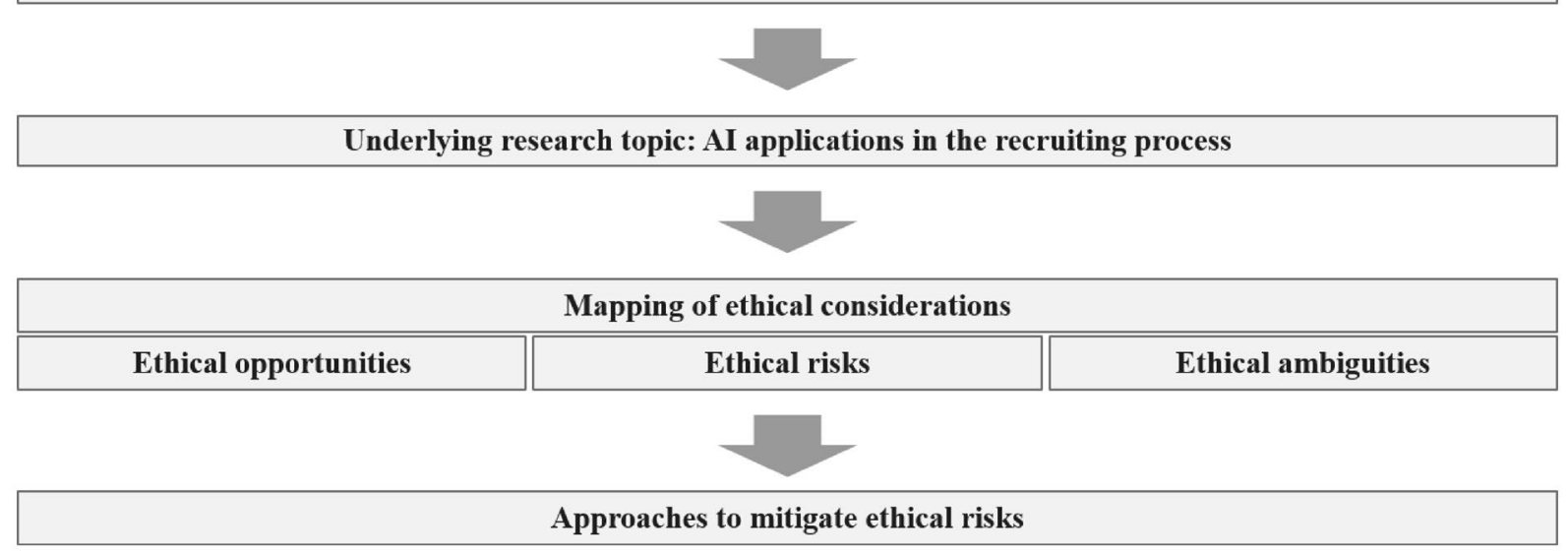

Fig. 1 Research design

Source Complete, Web of Science, and Scopus. Due to the novelty and interdisciplinarity of research on AI recruiting, we adopted a broad literature search strategy. We therefore decided in favor of openness of the sample and against an inclusion criterion such as publication in a top-tier journal of a specific field. Instead, we included all articles from academic peer-reviewed journals, conference proceedings, and practitioner-oriented articles (e.g., magazine articles) that study the ethicality of AI-powered practices in a company's recruiting and selection context. We ran the search by combining keywords from three topics: recruiting, ethics, and AI. Furthermore, we searched for articles in English without limiting the timespan. This initial search resulted in 756 hits after removing duplicates. The titles, abstracts, and full texts of all these articles were reviewed to determine the articles' relevance to our research scope, leading to 33 relevant articles. We then applied a backward search (by reviewing the references of the articles yielded from the keyword search) as well as a forward search (by reviewing additional sources that have cited the articles yielded from the keyword search), which resulted in a total of 51 distinct articles in scope for our review.

Our review excludes literature with a sole focus on a technical assessment of algorithmic fairness. Recently, a new body of literature emerged across such disciplines as law, policy, and computer science on fairness and bias in machine learning (ML) models, as well as their societal consequences (Barocas \& Selbst, 2016; Lepri et al., 2018). Many works within this stream have proposed different definitions of fairness and non-discrimination (e.g., Dwork et al., 2012; Hardt et al., 2016) and focus on technical options to identify, measure, and mitigate discrimination in ML models (e.g., Corbett-Davies et al., 2017; Zafar et al., 2017). Only if an article explicitly discussed the application field of recruiting, as well as ethical implications did we include it in our review. Furthermore, we set a narrow scope of AI-enabled recruiting and excluded all literature dealing with technology-enhanced recruiting practices in a broader sense. This literature stream had already emerged in the early 2000s and investigates perceptions of technology in personnel selection and job interviews (Wiechmann \& Ryan, 2003; Bauer et al., 2006; Chapman et al., 2003; see Blacksmith et al., 2016 for a meta-analysis). In various empirical studies, technology-mediated recruiting procedures, such as telephone and video interviews were investigated by testing the effects of technology-related factors on the interviews and the applicant reactions. For example, a couple of studies examined the fairness perceptions of applicants in online selection practices (Konradt et al., 2013; Thielsch et al., 2012). Nevertheless, we only included articles on recruiting practices that make use of AI techniques. Table 1 provides an overview of the data collection and selection criteria.

Table 1 Criteria for literature search and selection

\begin{tabular}{|c|c|}
\hline Search terms & $\begin{array}{l}\text { "Artificial Intelligence"; "AI"; "Algorithm*”; } \\
\text { "Machine Learning"; "Robot*” AND } \\
\text { "Ethic*”; "Moral*”; "Responsib*”; "CSR"; } \\
\text { "Philosoph*”; "Fair*”; "Bias*”; AND } \\
\text { "Recruit*”; "Hiring"; "Talent"; "Employee screen- } \\
\text { ing"; "Employee selection"; "Job interview*”; } \\
\text { "Applicant screening"; "Employment interview*” }\end{array}$ \\
\hline Search procedure & $\begin{array}{l}\text { Initial keyword search; backward search; forward } \\
\text { search }\end{array}$ \\
\hline Language & English \\
\hline Time frame & No limitation \\
\hline Databases & Business Source Complete, Web of Science, Scopus \\
\hline Inclusion & $\begin{array}{l}\text { Articles from journals or databases, primarily } \\
\text { related to ethicality of AI-enhanced recruiting, } \\
\text { accessible in full text }\end{array}$ \\
\hline Exclusion & $\begin{array}{l}\text { Letters to the editor, commentaries, interviews, } \\
\text { reviews, conference abstracts, and articles and } \\
\text { studies without direct relation to the ethicality } \\
\text { of AI-enabled recruiting }\end{array}$ \\
\hline
\end{tabular}




\section{Structural Analysis of the Literature and Categorization}

The chronological development of the identified literature underscores the novelty and increasing importance of our research topic in the last couple of years, with the first articles published in 2016. The 51 papers were published variously across 40 journals from different research fields, such as law, management, organizational psychology, robotics, and computer science. Table 2 shows the distribution of articles per journal and per year.
Based on our literature analysis, we identified five perspectives for categorizing the articles found: theoretical, practitioner, legal, technical, and descriptive. Two researchers independently performed the grouping according to these categories. ${ }^{1}$ In case of disagreement, agreement was reached through discussion. A comprehensive overview of the collected literature and its categorization is provided in Table 3.

Table 2 Distribution of articles per journal and year

\begin{tabular}{|c|c|c|c|c|c|}
\hline Journal Title & 20162017 & 2018 & 2019 & 2020 & Total \\
\hline Academic journals & & & & & 26 \\
\hline ABA Journal of Labor \& Employment Law & 1 & & & & 1 \\
\hline Annual Review of Org. Psychology \& Org. Behavior & & & & 1 & 1 \\
\hline Big-Data and Society & & 1 & & & 1 \\
\hline Business Horizons & & & 1 & & 1 \\
\hline Business \& Information Systems Engineering & & & & 1 & 1 \\
\hline California Law Review & 1 & & & & 1 \\
\hline California Management Review & & & 1 & & 1 \\
\hline Computers in Human Behavior & & 1 & 1 & & 2 \\
\hline Current Opinion in Behavioral Sciences & 1 & & & & 1 \\
\hline IBM Journal of Research and Development & & & 1 & & 1 \\
\hline IEEE Access & & & & 1 & 1 \\
\hline Industrial and Organizational Psychology & 1 & & & & 1 \\
\hline International Journal of Selection and Assessment & & & 1 & 1 & 2 \\
\hline Journal of Information Policy & & 1 & & & 1 \\
\hline Journal of Managerial Psychology & & & 1 & 1 & 2 \\
\hline Journal of Work and Organizational Psychology & & & 1 & & 1 \\
\hline Management Systems in Production Engineering & & & 1 & & 1 \\
\hline Online Information Review & & & 1 & & 1 \\
\hline Organizational Behavior and Human Decision Processes & & & & 1 & 1 \\
\hline Paladyn: Journal of Behavioral Robotics & & & & 1 & 1 \\
\hline Philosophy \& Technology & & & & 1 & 1 \\
\hline Saint Louis University Law Journal & & 1 & & & 1 \\
\hline William \& Mary Law Review & 1 & & & & 1 \\
\hline Practitioner journals & & & & & 15 \\
\hline CIO Magazine & 1 & & & & 1 \\
\hline Fast Company & & 1 & & & 1 \\
\hline Harvard Business Review & 1 & & 7 & & 8 \\
\hline Recruiter & & & & 1 & 1 \\
\hline SHRM & & 1 & & & 1 \\
\hline Training Journal & & & 1 & & 1 \\
\hline Workforce Solutions Review & & & 1 & & 1 \\
\hline Conference Proceedings & & & & & 11 \\
\hline AAMAS (Int. Conf. on Autonomous Agents and Multiagent Systems) & & & & 1 & 1 \\
\hline
\end{tabular}


Table 2 (continued)

Journal Title $20162017 \quad 2018 \quad 20192020$ Total

Academy of Management Proceedings

AIES (AAAI/ACM Conf. on AI, Ethics, and Society)

FAT* (Conf. on Fairness, Accountability, and Transparency)

BESC (Int. Conf. on Behavioral, Economic Advance in Behavioral, Economic, and Sociocultural Computing)

CVPRW (Conf. on Computer Vision and Pattern Recognition Workshops)

ICIS (Int. Conf. on Information Systems)

IFIP Advances in Information \& Communication Technology

IMC (Int. Mgmt. Conf. on Mgmt. Strategies for High Performance)

International Symposium on Technology and Society

Total

$20162017 \quad 2018 \quad 20192020 \quad$ Total

1

1

1

22

11

1

1

1

11

$1 \quad 1$

$\begin{array}{llllll}4 & 5 & 7 & 22 & 13 & 51\end{array}$ 


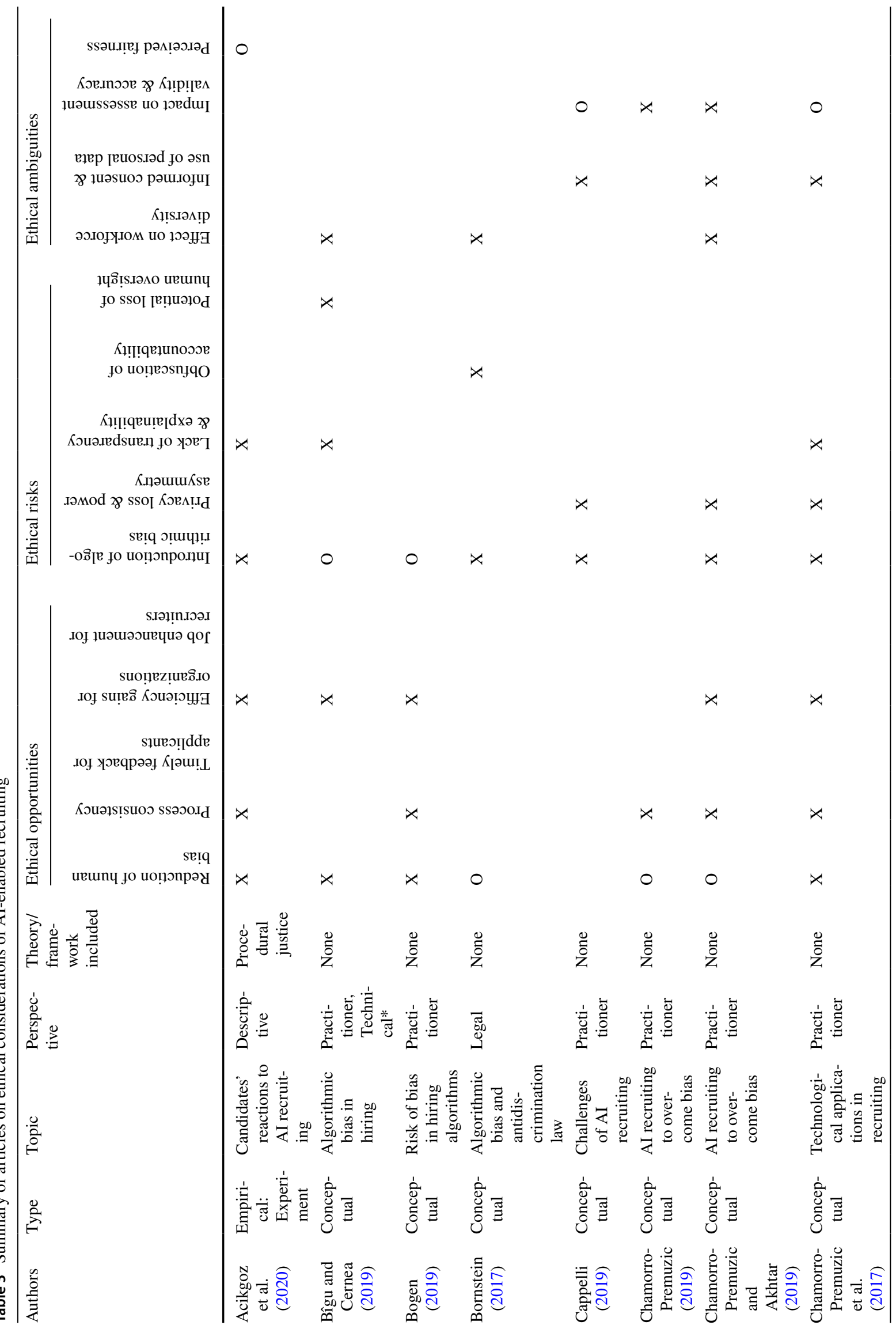




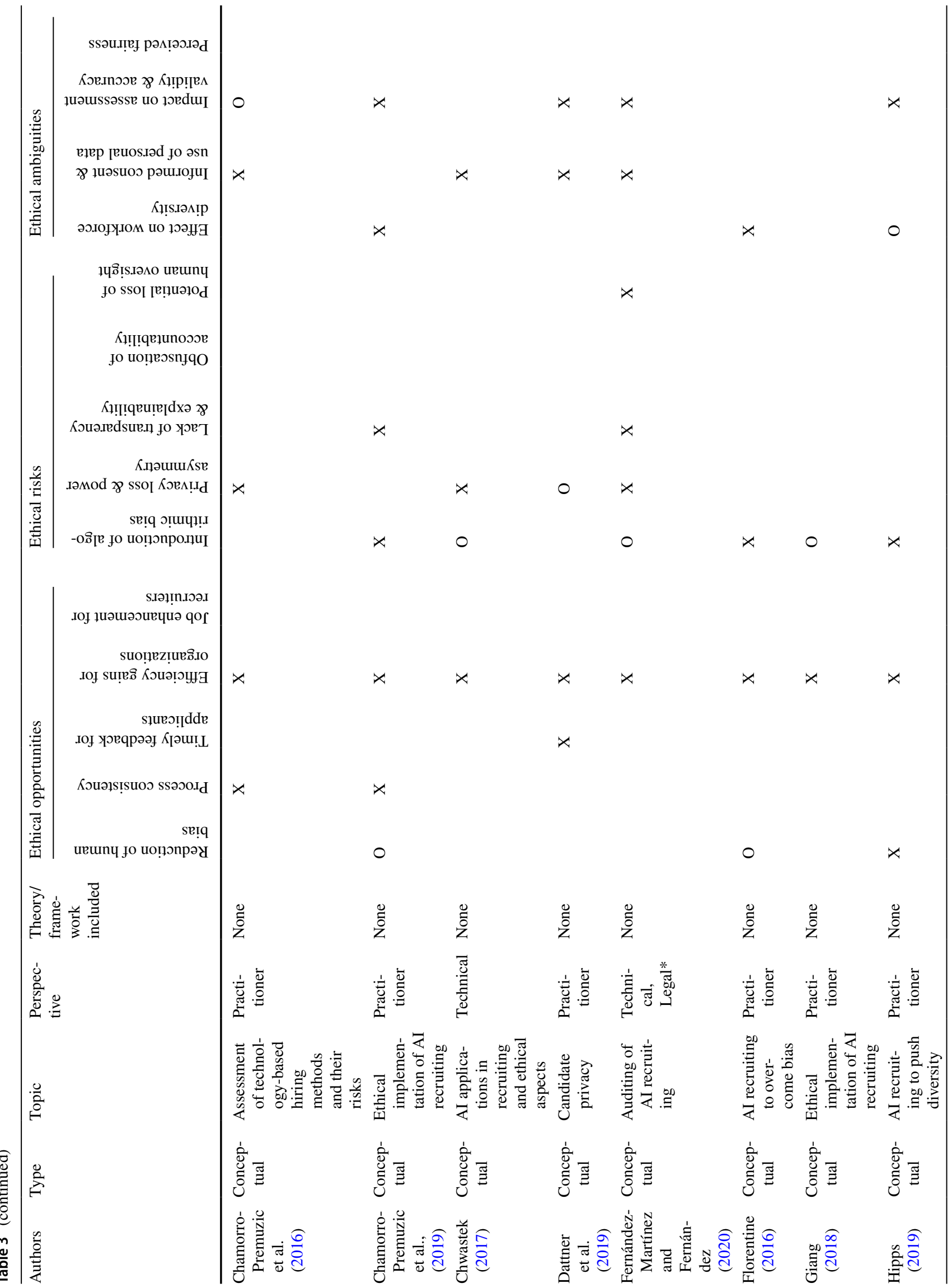




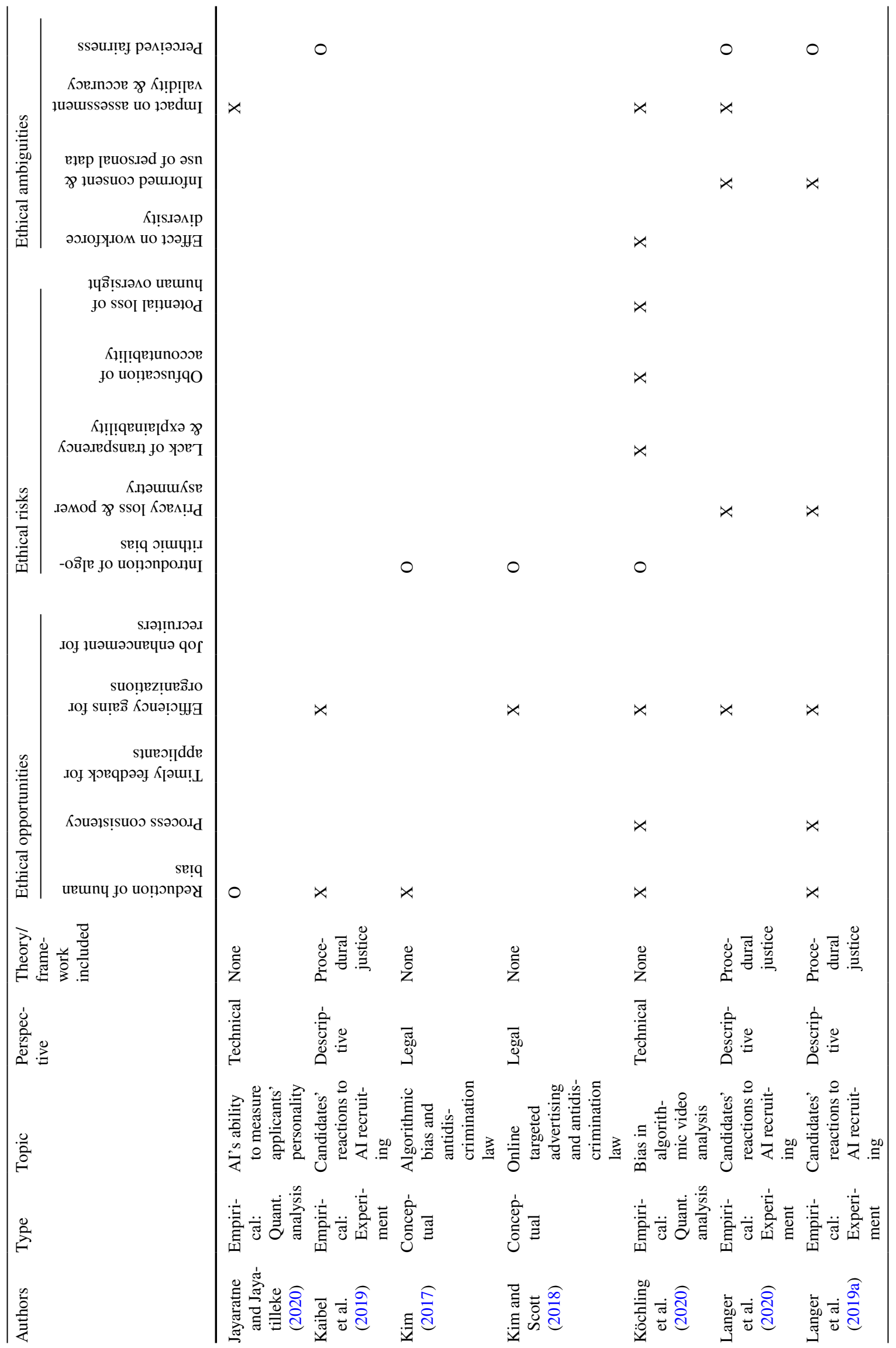




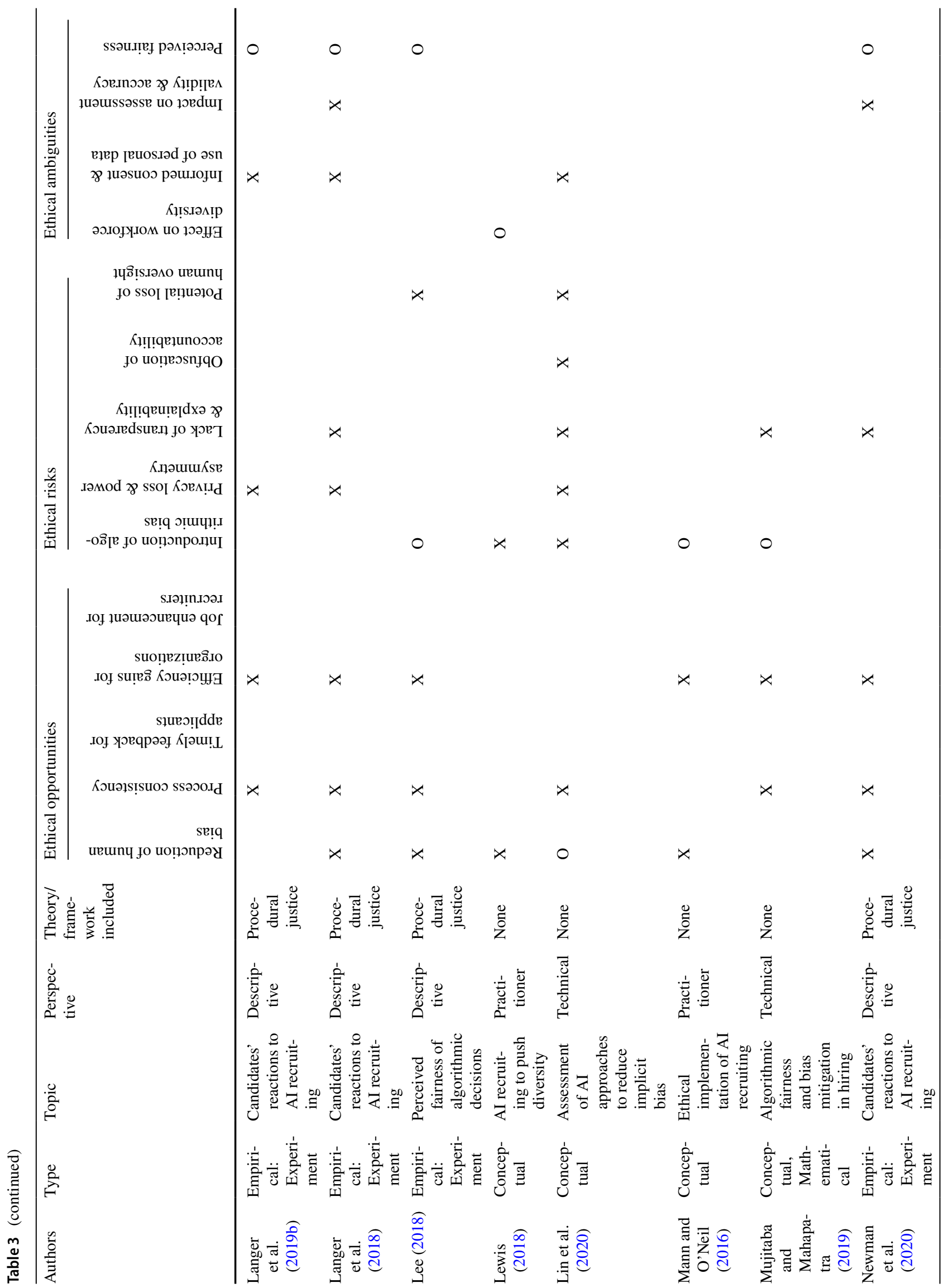




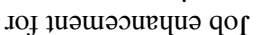

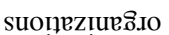

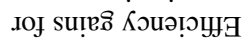
słueว!̣ .оу уәвqрәәу К[әш!. Кวนวฺรเงนoว ssəวo.I

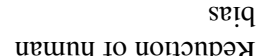
ते 峁 Z ¿̊

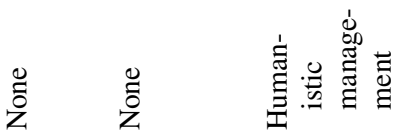

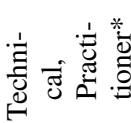

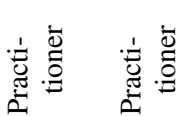
咅

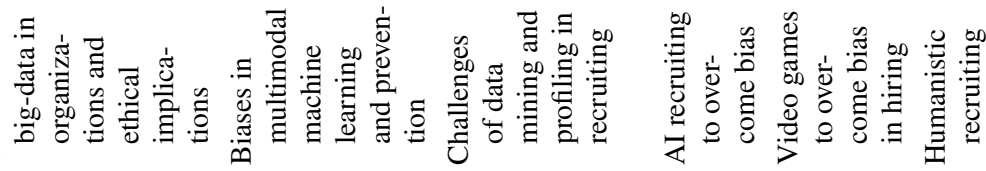

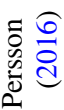

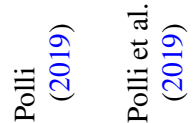

悹 


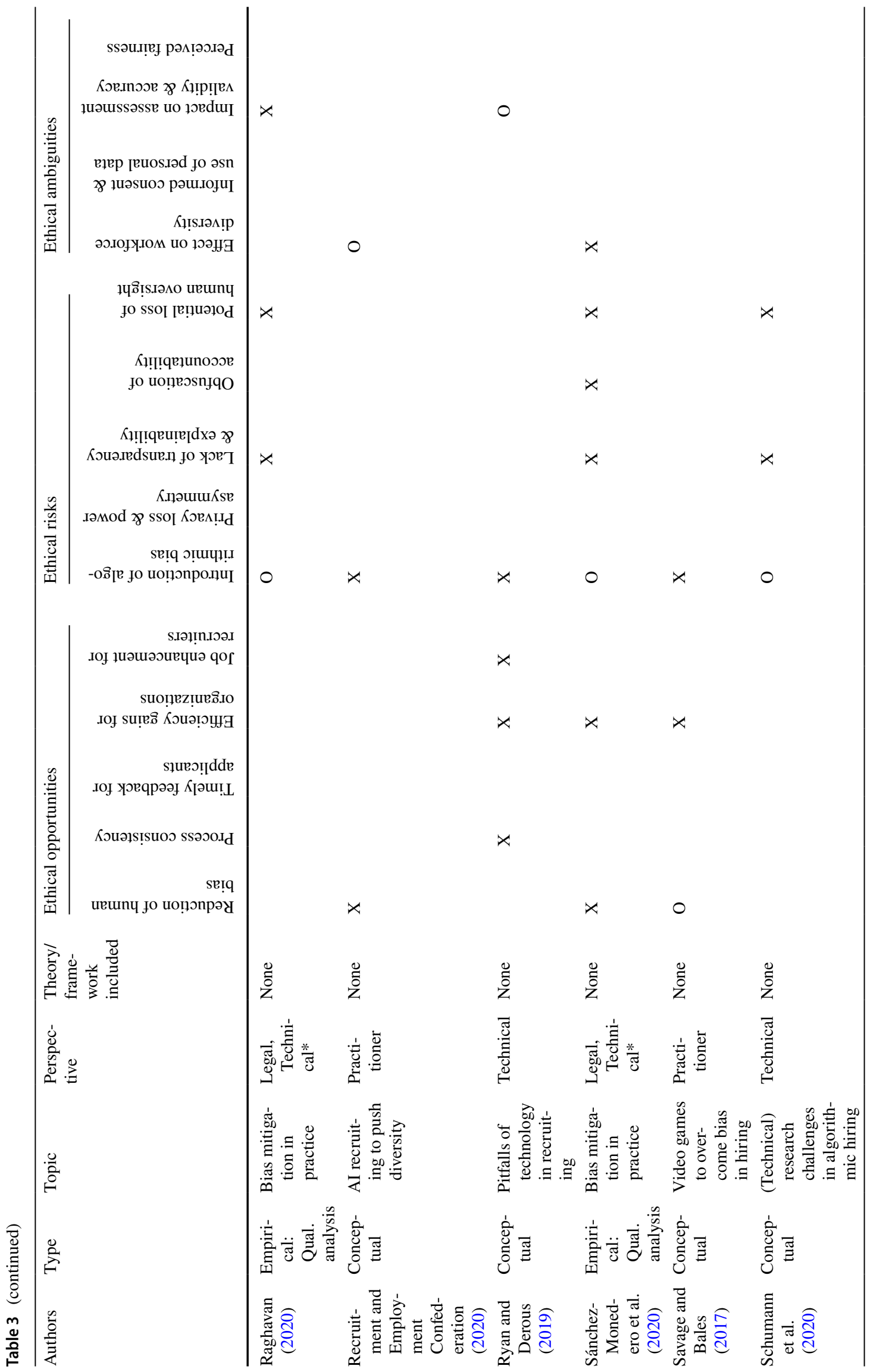




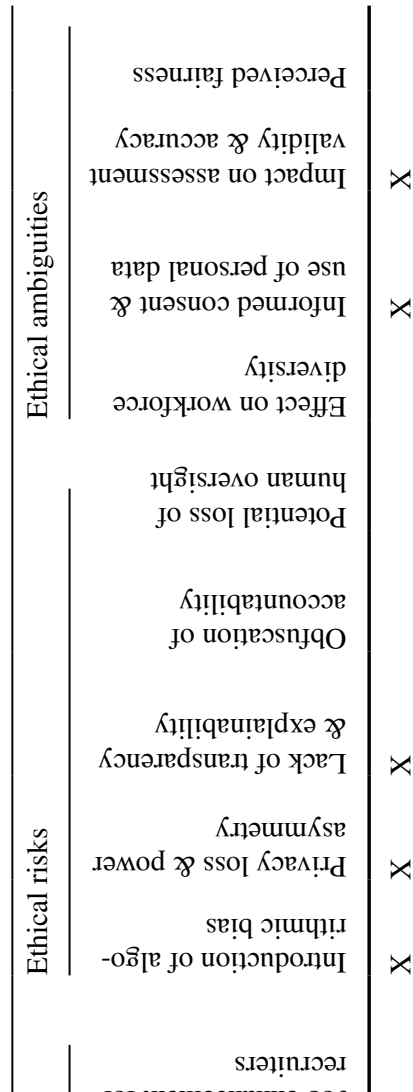

.I0 1นวшәวนечนว qo[

suọ̣pz!̣ueo̊.ıo IOJ su!̣ภิ Кวนวฺฺษ

słues! [dde

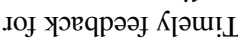

Кวuəฺฺฺฺsuoว ssəวo.Id

जв!̣

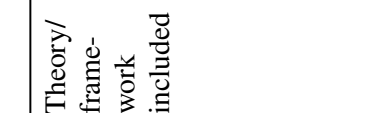

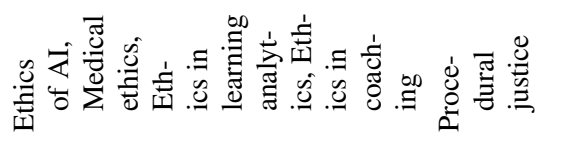

ڤั๊

常

Z̃

离

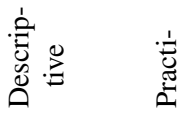

窎

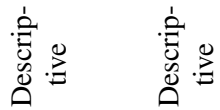

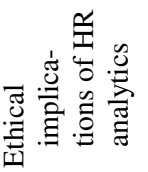

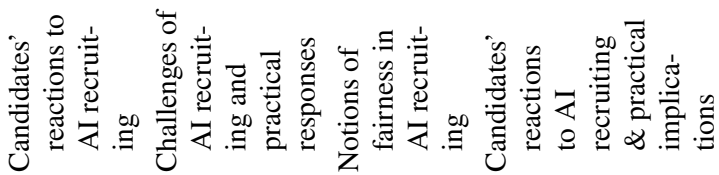

这

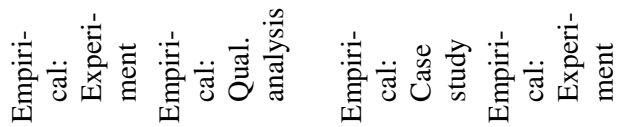

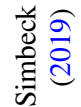

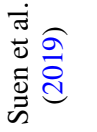

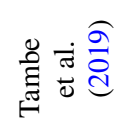

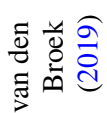

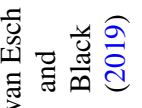




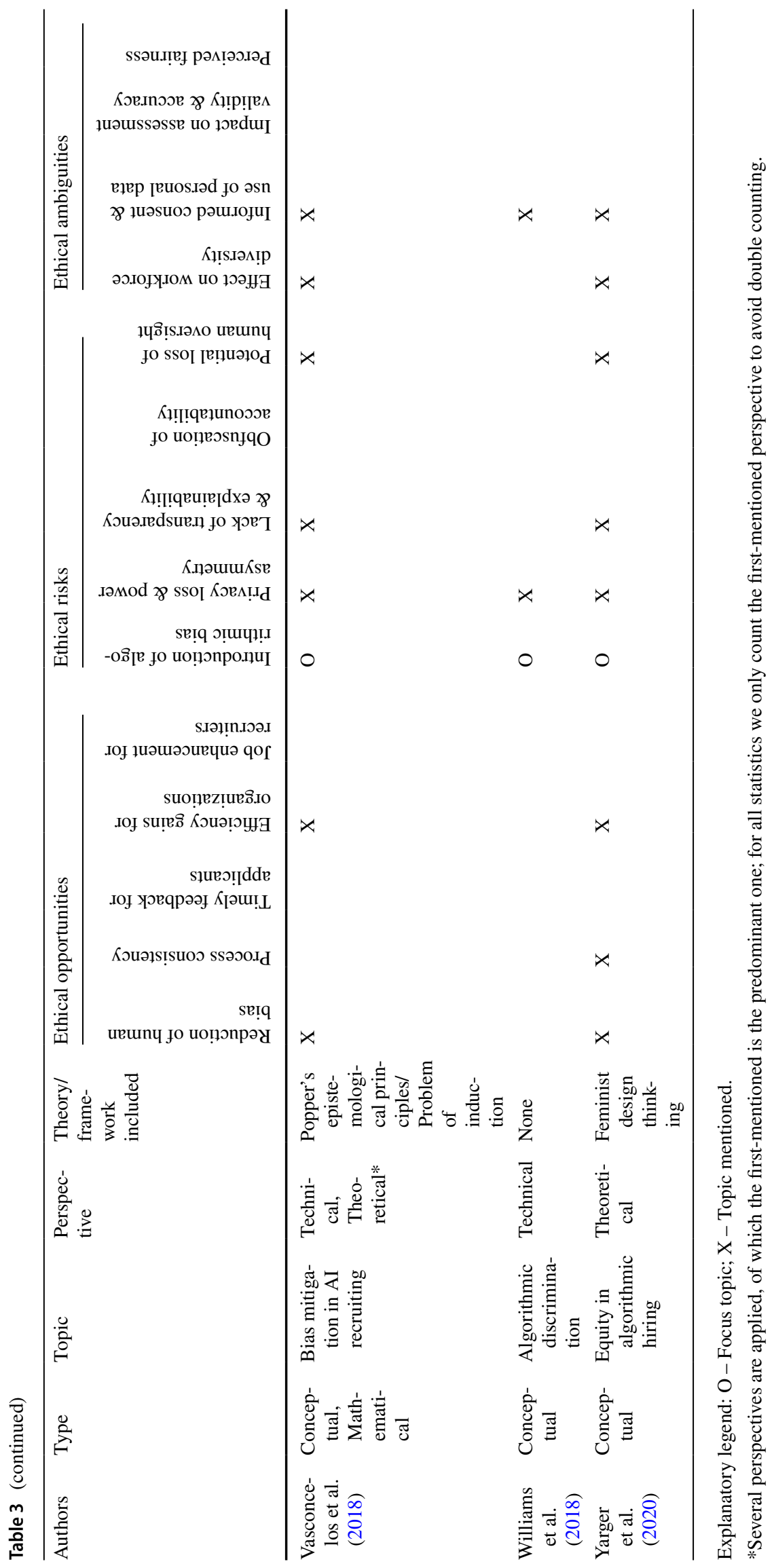


Overall, the articles falling into the practitioner perspective clearly constitute the majority of extant research on the ethicality of AI recruiting (39\%), followed by the technical, descriptive, and legal perspectives (24\%, 22\%, and $10 \%)$, and lastly, the theoretical perspective $(6 \%)$.

\section{Findings}

\section{Perspectives on the Ethicality of AI-Enabled Recruiting and Selection}

We start by reviewing the different perspectives from which AI-enabled recruiting and selection practices are investigated and ethical considerations are articulated.

\section{Theoretical Perspective}

The first group of papers assessed AI-powered recruiting practices from an ethics theory perspective. We identified three articles that applied a theoretical framework to AI recruiting and thereby provide a theoretical foundation for discussion: First, Simbeck (2019) referred to ethical frameworks from other disciplines, such as medicine, robotics, and AI, and applied them to the HR context. She proposed the transfer of key ethical concepts from the other fields that should be implemented when applying new AI technologies in HR analytics. She identified five key ethical principles: privacy and confidentiality, opportunity to opt out, institutional review, transparency, and respect for the dynamic nature of personal development.

Second, Yarger et al. (2020) referred to feminist thinking and methods, arguing that these should guide the design of AI hiring systems. Feminist approaches shed light on the extent to which algorithms may perpetuate disadvantage for historically marginalized groups when equity is not considered in their design. The authors presented a feminist design justice framework, which includes prompts that commit the architects of AI systems to engage with the design process in ways that support an ethic of equity.

Third, Rąb-Kettler and Lehnervp (2019) assessed AI recruiting from a humanistic perspective, in which people were placed at the center. The authors presented humanistic recruiting as an answer to the current technological developments. They argued that technology and automation can be implemented in a way that improves the experience for both the recruiters and candidates in the process. They concluded that both humanistic insight and sophisticated technology are important to adjust to today's dynamic reality. Reviewing these three theoretical papers reveals that a detailed assessment of AI recruiting from the standpoint of one of the traditional ethics theories, such as utilitarianism or deontology, and a discussion of potential implications for the hiring practice has not been done yet.

\section{Practitioner Perspective}

The second and largest category of papers assumed a practiceoriented perspective and focused on implications that are most relevant for managers and corporations. Most of the identified papers fall into this group, the common aim of which was to raise practitioners' awareness of the strengths and limitations of AI technologies implemented in the recruiting process. From an experience-based perspective, some papers (Florentine, 2016; Polli et al., 2019) underlined the problematic nature of traditional candidate assessment methods and presented the use of $\mathrm{AI}$ as a promising alternative; others (Bogen, 2019; Dattner et al., 2019) rather warned of AI-powered hiring practices by raising many yet-unanswered questions about their accuracy, as well as the ethical, legal and privacy implications that they introduce. Furthermore, some papers (Bîgu \& Cernea, 2019; Chamorro-Premuzic et al., 2019; Giang, 2018; Mann \& O’Neil, 2016) provided practical recommendations for managers on how to ethically implement AI for recruiting, aiming to guide organizations to take the right steps and make the right investments.

\section{Legal Perspective}

The third group of papers looked at AI recruiting from a legal viewpoint. The importance of employment decisions to individuals, as well as to broader society, has led to the design of an extensive legal framework to guide these decisions. For example, in the US, Title VII of the Civil Rights Act protects people from discrimination in any employment decision that would result in disparate treatment ${ }^{2}$ or disparate impact ${ }^{3}$. It also assigns liability and legal responsibility to employers to ensure that the tools used do not create such results. However, the identified literature (Bornstein, 2017; Kim, 2017; Kim \& Scott, 2018) has claimed that, so far, the law of Title VII lags behind current scientific knowledge and modern business practices: Kim and Scott (2018) discussed that targeted advertising may result in unfair exclusions that are not covered by current law, Bornstein (2017) argued that current regulation does not go far enough and argued for liability when an employer acts with reckless disregard for the consequences of implicit bias in employment decisions, and Kim (2017) claimed that Title VII should be broadened, requiring employers to prove that the data created by their algorithms are accurate and do not discriminate, instead of requiring victims of discrimination to prove its occurrence. We further identified two qualitative analyses that embraced both a legal and a technical perspective, while investigating how bias mitigation methods are used in practice. While Raghavan et al. (2020) evaluated the efforts of AI software vendors to mitigate bias, focusing on the employment laws in the US, Sánchez-Monedero et al. (2020) analyzed three recruiting software vendors from the perspective of UK law, addressing concerns over both discrimination and data protection. 


\section{Technical Perspective}

Moreover, we identified a group of articles that established ethical considerations on AI recruiting, while taking a technical perspective. Some papers (Chwastek, 2017; Köchling et al., 2020; Lin et al., 2020; Mujtaba \& Mahapatra, 2019; Persson, 2016; Williams et al., 2018) explained emerging ethical problems by looking at the mechanisms of algorithms used. Others (Fernández-Martínez \& Fernández, 2020; Pena et al., 2020; Vasconcelos et al., 2018) presented technical solutions to implement ethical principles into algorithmic code or design. For instance, Fernández-Martínez and Fernández (2020) found that there is a lack of regulation and a need for external and neutral auditing of the used AI technologies, and consequently, they presented a multi-agent software architecture to support auditing the recruiting processes. Furthermore, Vasconcelos et al. (2018) proposed a computational framework to mitigate discrimination and unfairness caused by bias in AI systems, inspired by epistemological principles. Lastly, one paper (Schumann et al., 2020) outlined several technical challenges for future research in algorithmic hiring that must be overcome to make it fairer and more intelligible.

\section{Descriptive Perspective}

Covering the field of descriptive ethics, the last category comprises several experimental studies (e.g., Langer et al., 2018; Lee, 2018; van Esch \& Black, 2019), as well as a case study (van den Broek et al., 2019) that assessed people's reactions to AI-powered recruiting practices. A couple of studies compared applicants' fairness perceptions of AIenabled interviews vs. traditional interviews with a human recruiter, revealing contrasting findings. Whereas a group of papers (Acikgoz et al., 2020; Lee, 2018; Newman et al., 2020) found that people perceived algorithm-driven decisions as less fair than human-made decisions, another group of papers (Langer et al., 2019a, 2019b, 2020; Suen et al., 2019) found no difference in fairness perception between decisions made by an AI or a human. Other studies (Gelles et al., 2018; Kaibel et al., 2019; Langer et al., 2018; van Esch \& Black, 2019) examined different contextual and procedural factors, such as the level of information given to applicants regarding the used AI or the level of computer experience of applicants, and how they affect applicant reactions to the use of $\mathrm{AI}$ in hiring.

In summary, this overview attests to the overall heterogeneous perspectives applied to ethical considerations of AIbased recruiting and selection. It also reveals that only a few theoretical articles exist, and that extant literature is rather practitioner oriented.

\section{Underlying Research Topic: Al Applications in the Recruiting and Selection Process}

In the following, we provide an overview of AI applications used in the recruiting and selection process and addressed in the identified literature. An understanding of where AIpowered tools and practices are applied can assist in understanding where ethical opportunities and risks may arise. Our review shows that AI-enabled practices are relevant in each stage of the recruiting process and can include different types of AI and algorithms. Table 4 gives an overview of the different AI applications across the recruiting and selection stages: outreach, screening, assessment, and facilitation, which we further expand on below. 
Table 4 AI applications per recruiting stage

\begin{tabular}{|c|c|c|c|c|}
\hline Stage & Outreach & Screening & Assessment & Facilitation \\
\hline Objective & $\begin{array}{l}\text { Identify possible candi- } \\
\text { dates \& persuade them } \\
\text { to apply }\end{array}$ & $\begin{array}{l}\text { Derive shortlist of most } \\
\text { promising candidates }\end{array}$ & $\begin{array}{l}\text { Identify which candidate is most } \\
\text { appropriate for the job }\end{array}$ & $\begin{array}{l}\text { Coordinate with applicants through- } \\
\text { out the process }\end{array}$ \\
\hline $\begin{array}{l}\text { AI applica- } \\
\text { tions }\end{array}$ & $\begin{array}{l}\text { Formulation of job ads } \\
\text { (e.g., gender-neutral } \\
\text { wording) } \\
\text { Targeted advertisement of } \\
\text { open positions (e.g., via } \\
\text { social media) } \\
\text { Notification of job seekers } \\
\text { Identification of active or } \\
\text { passive candidates (e.g., } \\
\text { via LinkedIn or ATS }{ }^{\text {a }} \text { ) }\end{array}$ & $\begin{array}{l}\text { Scanning of resumes } \\
\text { (beyond keywords) to } \\
\text { score or rank candidates } \\
\text { Matching of candidates \& } \\
\text { job openings to identify } \\
\text { best fit }\end{array}$ & $\begin{array}{l}\text { Analysis of video interviews } \\
\text { with AI technology (voice/face } \\
\text { recognition) } \\
\text { Simulation/games/tests to assess } \\
\text { certain skills, capabilities and } \\
\text { traits } \\
\text { Scraping \& analytics of social } \\
\text { media postings for psychological } \\
\text { profiles } \\
\text { Linguistic analysis of writing } \\
\text { samples \& web activity }\end{array}$ & $\begin{array}{l}\text { Use of NLP } \text { to parse CVs \& extract }^{\mathrm{b}} \text { relevant information to fill-in } \\
\text { application forms automatically } \\
\text { Transparency on where applicants } \\
\text { stand in the process \& elucidation } \\
\text { of next steps } \\
\text { Scheduling of interviews \& sending } \\
\text { of job offers } \\
\text { Communication with applicants \& } \\
\text { answering of questions by chatbot }\end{array}$ \\
\hline
\end{tabular}

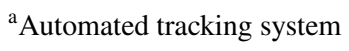

${ }^{\mathrm{b}}$ Natural language processing

\section{Outreach}

Several articles deal with AI technologies applied in the outreach stage, in which businesses try to detect talent and attract applicants. By leveraging algorithms for targeted communication across online platforms and social media or for the automated notification of job seekers, companies can expand their outreach to potential candidates (Bogen, 2019). Furthermore, AI bots are used to identify the pool of active and passive candidates (e.g., via LinkedIn) or to (re-)discover top talents in the pool of former candidates via their internal automated tracking system (ATS) (van Esch \& Black, 2019). Sometimes, the challenge is not just finding the right candidates but persuading them to apply via appealing job descriptions. AI software vendors, such as Textio, use $\mathrm{AI}$ in the form of text-mining techniques to predict the attractiveness of a job listing based on the hiring outcomes of several millions of job posts. The software thereby scans the job ad for key phrases that will statistically impact its performance. Additionally, a tone meter can determine whether the overall tone of the writing is likely to attract more men or more women and make suggestions on how to improve the inclusiveness of the language used (Lewis, 2018; Yarger et al., 2020). This is how AI can help businesses de-bias the wording of job ads, making them gender neutral to attract a diverse pool of applicants, or customize them for a specific target group (Rąb-Kettler \& Lehnervp, 2019).

\section{Screening}

Notably, most articles that deal with the ethicality of AI recruiting focus on the application of AI technology in an initial resume screening. AI systems are used to filter applicants to derive a shortlist and a ranking of the most promising candidates (Bornstein, 2017; Fernández-Martínez \& Fernández, 2020; Vasconcelos et al., 2018). For many years, companies have used traditional algorithms to scan resumes for preselected key words or phrases; however, today's AI technology goes beyond that. Now, chatbots and resume-parsing tools look for semantic matches and related terms determining a candidate's qualification. Other tools go even further and use ML to make predictions about a candidate's future job performance based on signals related to tenure or productivity, or the absence of signals related to tardiness or disciplinary action (Bogen, 2019). Based on the initial screening, algorithms can also suggest the best matching job opening for a given candidate (Rąb-Kettler $\&$ Lehnervp, 2019). These screening tools are considered highly efficient to streamline the process, especially for top employers who receive huge numbers of applications for each open position; however, concerns have been raised that highly qualified applicants may be overlooked (Persson, 2016).

\section{Assessment}

Although screening algorithms are not new in practice, there has been a recent trend toward video-interview analysis in recruiting. In such structured video interviews, AI technology replaces a human interviewer and asks the candidate a short set of predetermined questions (Chamorro-Premuzic et al., 2016; Fernández-Martínez \& Fernández, 2020). Moreover, the AI technology can not only evaluate the actual responses, but also make use of audio and facial recognition software to analyze additional factors such as the tone of voice, microfacial movements, and emotions to provide insights on certain personality traits and competencies 
(Köchling et al., 2020; Tambe et al., 2019; van Esch \& Black, 2019).

Besides interviews, AI-powered skill tests, simulations, and neuroscience video games are used to assess further qualities, for example, applicants' risk attitude, planning abilities, persistence or motivation. Thereby, target variables need not be predefined by the company (Giang, 2018; Polli et al., 2019; Raghavan et al., 2020), but ML algorithms can analyze the data of a company's current top performers and derive which applicant characteristics and skills have been associated with better job performance (Tambe et al., 2019). In this way, data-driven assessment tools have changed talent signals and the criteria by which candidates are evaluated (Chamorro-Premuzic et al., 2016). For example, the software vendor Pymetrics uses ML and psychometric training data based on current top performers to predict an applicant's fit for a specific role. To this end, first, the top-performing incumbent employees in that role play a series of online games, which are gamified assessments that measure numerous cognitive and social traits. The data collected from these games are then used to establish a "success profile" for the job at hand. Second, the candidates applying to the job play the same games, and the ML model predicts their likelihood of success in the role (Polli et al., 2019).

Other software vendors offer AI technologies that analyze a person's digital records such as social media posts to construct a psychological profile of a candidate. Based on linguistic analyses of candidates' Web activities, new technologies infer talent, personality, and other important individual differences and compare them against the culture of the hiring company (e.g., Chamorro-Premuzic et al., 2016, 2017; Vasconcelos et al., 2018).

\section{Facilitation}

Finally, AI is used to facilitate the recruiting process, taking over administrative tasks. For instance, AI tools address the problem of long online questionnaires for applicants via natural language processing (NLP) techniques. These are used to parse unstructured documents, such as candidates' CVs, and extract relevant information to automatically complete a company's application form (Chwastek, 2017). Furthermore, AI-powered assistants can be used to interact and communicate with candidates: They can guide candidates through the different steps of the recruitment process, from answering company and process-related questions to scheduling interviews (Rąb-Kettler \& Lehnervp, 2019; van Esch \& Black, 2019). Today, many companies also use programs to create offers automatically and have them signed electronically (Sánchez-Monedero et al., 2020).

\section{Mapping of Ethical Considerations}

This rise of new AI recruiting practices comes with new ethical quandaries for organizations and society. In what follows, we examine extant research literature and map the ethical considerations established. This mapping of ethical considerations can be understood as a summary of areas in which society may have ethical concerns about the use of AI, which is derived from extant literature. In mapping the ethical considerations, we distinguish between aspects that are, on the one hand, clearly characterized as morally good and thus as ethical opportunities, and on the other hand, aspects that are clearly characterized as morally bad and thus ethical risks. In addition, we outline issues that are controversially discussed in the literature and thus reflect ethical ambiguities that require deeper exploration. Table 5 provides a structured overview of this ethical evaluation.

\section{Human and Algorithmic Bias}

The most-discussed topic in extant literature on AI-enabled recruiting is the occurrence of bias. Although there is broad agreement that the practices currently in place are far from effective and unbiased (e.g., Chamorro-Premuzic \& Akhtar, 2019; Persson, 2016; Polli, 2019), there are two differing ways, in which AI-powered tools may effect the scope of bias.

Table 5 Overview of ethical evaluation: Ethical opportunities, risks and ambiguities

\begin{tabular}{lll}
\hline Ethical opportunities & Ethical risks & Ethical ambiguities \\
\hline Reduction of human bias & Introduction of algorithmic bias & Effect on workforce diversity \\
Process consistency & Privacy loss \& power asymmetry & Informed consent \& use of personal data \\
Timely feedback for applicants & Lack of transparency \& explainability & Impact on assessment validity \& accuracy \\
Efficiency gains for organizations & Obfuscation of accountability & Perceived fairness \\
Job enhancement for recruiters & Potential loss of human oversight & \\
\hline
\end{tabular}


On the one hand, the use of AI may reduce human bias in different stages of the recruiting process and should therefore be considered a huge ethical opportunity (e.g., Chamorro-Premuzic \& Akhtar, 2019; Savage \& Bales, 2017). In the outreach stage, AI can address bias in the form of gendered language in job descriptions that dissuades certain candidates from applying for a role by creating inclusive job descriptions (Mann \& O'Neil, 2016; Recruitment \& Employment Confederation, 2020). In the screening procedure, subjectivity can be reduced by using algorithms that screen all applicants against the same criteria. $\mathrm{AI}$ is thereby able to assess the entire pipeline of candidates rather than forcing time-constrained humans to shrink the pool from the start, based on a biased process. Instead, AI can shrink the initial pipeline so a recruiter with a constrained capacity can manually handle it (Polli, 2019). Especially in the assessment stage, the use of AI technology can remove human bias from the process - or at least reduce it substantially. Human intuition can be very good and accurate, but it is nevertheless based on subjective value assessment (Persson, 2016). In contrast, via a digital interview or a video game assessment, AI automatically captures many data points of the applicants' behavior, such as what they say, their language use or their body language, for an objective, data-driven assessment of personality (Jayaratne \& Jayatilleke, 2020). Moreover, human bias (e.g., related to applicants' physical appearance or other attributes) can be reduced, as AI can be taught to ignore people's personal attributes and focus only on specified skills and behaviors (e.g., Bîgu \& Cernea, 2019; Chamorro-Premuzic \& Akhtar, 2019; FernándezMartínez \& Fernández, 2020). Lastly, human bias can be removed from the process, as the required skills and qualities for successful candidates are not determined by bias-prone intuitions from recruiters, but based on analyzing the characteristics of the company's top performers (Lin et al., 2020).

On the other hand, AI-enabled recruiting also bears the risk of introducing different types of algorithmic bias (e.g., Bogen, 2019; Yarger et al., 2020). Yarger et al. (2020) cited three factors that may lead to biased decisions: bias in the model design principles, bias in the feature selection, and bias in the training data. A biased design, for example, may be manifested in online job platforms that make superficial predictions, not focusing on who will be successful in the role, but on who is most likely to click on the job ad. This can lead to a reinforcement of gender and racial stereotypes. A study found that targeted ads on Facebook for supermarket cashier positions were shown to an audience of $85 \%$ women, indicating that adverse impact can also occur in sourcing algorithms (Bogen, 2019). Moreover, critics are concerned that algorithms derived from information about current employees will unintentionally discriminate against underrepresented groups if existing employees are not proportionately representative of the broader application pool; this would constitute a case of biased training data (Kim, 2017). A known example from practice is the Amazon case, in which a hiring algorithm (in test mode) discriminated against women, assigning lower scores to resumes of women when ranking candidates. The algorithm was trained on data of current top performers, of which the majority were male. Thus, the algorithm penalized female attributes (e.g., Mujtaba \& Mahapatra, 2019). In all these cases, algorithms can introduce bias and even magnify discrimination, affecting entire classes of individuals (Bogen, 2019; Tambe et al., 2019). The occurring discrimination may thereby be direct or indirect via proxy attributes. In the latter case, a protected group (e.g., a specific race) is discriminated against but based on legitimate grounds (e.g., a zip code) (Bîgu \& Cernea, 2019; Fernández-Martínez \& Fernández, 2020).

Proponents of AI recruiting tools admit that adverse impact can occur; however, they state that, compared with human biases, algorithmic biases are much easier to detect and remove (Florentine, 2016; Polli, 2019). Often, the fear of biased AI ignores the fact that the original source of algorithmic bias is the human behavior it is simulating (e.g., the biased data set used to train the algorithm). Thus, if people criticize what the AI is doing, they should criticize human behavior even more because AI is purely learning from humans (Polli, 2019).

Although there is an ongoing debate on the potential occurrence of algorithmic bias in AI recruiting, there is no ambiguity on the topic itself but general agreement that all kinds of bias and discrimination should be prevented. Therefore, AI recruiting can be classified as ethically preferable, as long as it seeks to reduce interpersonal bias in the process. However, current research suggests that the usage of AI can reduce bias but is never completely free of bias and carries the risk of algorithmic discrimination, even without bad intentions on the part of the programmers, which should be morally denounced. Thus, technical due diligence regarding algorithmic design and implementation is crucial to keep this risk low (see Sect. 3.4).

\section{Effect on Workforce Diversity}

A topic closely related to the occurrence of bias in the selection process is its impact on diversity: On the one hand, a reduction in human bias could lead to diversification of a company's workforce (Chamorro-Premuzic \& Akhtar, 2019; Recruitment \& Employment Confederation, 2020). For example, the use of bias-neutral job posts created through AI may result in a more diverse pool of applicants (Lewis, 2018). Furthermore, the data-driven assessment leads to hiring of "nontraditional" candidates who might typically not make it through a hiring process (e.g., from a non-elite college, but with other strong skills). In this way, AI-enhanced recruiting tools can provide people from a wider range of 
socioeconomic backgrounds access to better jobs, expanding diversity, and socioeconomic inclusion (e.g., Florentine, 2016; Hipps, 2019). Moreover, case studies have shown that, for example, the aforementioned AI-powered video games by Pymetrics have a clear positive impact on companies' gender diversity (Polli et al., 2019).

On the other hand, a systematic bias through AI could result in more homogeneity in organizations (ChamorroPremuzic et al., 2019; Vasconcelos et al., 2018; Yarger et al., 2020). As a single decision-making algorithm, which selects candidates based on certain profiles and traits, replaces several human decision makers with potentially differing views, this may also imply a loss in diversity (Vasconcelos et al., 2018; van den Broek et al., 2019; Bîgu \& Cernea, 2019). Further, Fernández-Martínez and Fernández (2020) warned that the use of AI leads to increased racial bias: Given that emotional recognition software may not consider different intonations in different languages or that emotions are differently expressed in different cultures, it may systematically disadvantage specific races or ethnic groups, which could lead to a decrease in workforce diversity.

This research question about the influence of AI on diversity has also been discussed in general diversity scholarship. Ozkazanc-Pan (2019) outlined how advanced technological shifts impact diversity scholarship, underlining the importance of bias, ethical considerations and digital inequalities in this context. She also thereby referred to the recruiting context and, for example, pointed out how the creation of employee profiles that are based on behavioral preferences, when not implemented carefully, can lead to HR managers hiring the same groups over and over again, which can hinder a company's diversity efforts.

Overall, there is no clear understanding of what impact the use of AI has on the diversity of corporate workforces, but the topic is controversially discussed in extant literature. Therefore, relevant empirical studies would be desirable in future. It must be noted that diversity is related to, but different from, non-discrimination, and more textured efforts are needed to explore the balance between diversity and non-discrimination (Schumann et al., 2020). An interesting question in this context may be whether it is ethical to promote diversity even if it discriminates against historically advantaged groups.

\section{Privacy and Informed Consent}

Another ethical consideration raised is the concept of privacy and informed consent. In this context, businesses must account for government regulations, which differ across countries. The European General Data Protection Regulation (GDPR), which came into effect in May 2018, is one of the strictest. It aims to protect EU citizens' rights by regulating how to collect, store, and process personal data and requires informed consent for any personal data processing (i.e., applicants must have the opportunity to agree or not agree to the use of their data). However, the informed consent requirement is not yet well implemented in the bigdata and AI-regulation context, rendering the protection of personal privacy an ethical challenge (Oswald et al., 2020). An ethical dilemma emerges at this point as applicants in the job market generally hold less power than employers. Even if applicants are informed enough to consent to the process, they may not be able to opt out without being disadvantaged in the process. It is therefore difficult to give explicit consent in the context of hiring anyhow (Sánchez-Monedero et al., 2020).

Moreover, there is active debate about the extent to which it is ethically appropriate to use social media information for personnel selection purposes (Chamorro-Premuzic et al., 2016; Oswald et al., 2020). Legally, social media content is public data, but it is questionable whether it is ethical to mine social media data for hiring purposes when users generally use those platforms for other purposes and may not have provided their consent for data analysis (Dattner et al., 2019; Tambe et al., 2019). Also, the extent to which social media posts are a valid and reliable indicator of personality or job performance is doubtful (Vasconcelos et al., 2018; Yarger et al., 2020). Chamorro-Premuzic et al. (2016) argued that it is naive to expect online profiles to be more authentic than resumes, but they can offer a wider set of behavioral samples. Prior empirical findings on the validity of social media data have been mixed (Ryan \& Derous, 2019). Whereas some studies found connections to job performance (e.g., Kluemper et al., 2012), van Iddekinge et al. (2016) showed that recruiter ratings of applicants' Facebook information were unrelated to their subsequent job performance and lead to subgroup differences, by favoring female and Caucasian applicants. This discussion on the use of social media information in the hiring context is not new and only connected to the use of AI. A study in Sweden showed that at least half of the interviewed recruiters had scanned applicant social media profiles themselves at some point before hiring (Persson, 2016). However, the new AI techniques make the analysis of social media profiles easier and even more tempting.

There are further AI-enabled ways to discern applicants' private information indirectly. For example, image and voice recognition techniques can predict applicants' sexual orientation, race, and age, as well as their physical attractiveness (Chamorro-Premuzic et al., 2016; Dattner et al., 2019). Other prediction algorithms may forecast who is more likely to become pregnant (Oswald et al., 2020; Simbeck, 2019). This greater access to candidates' personal attributes can not only increase the risk of misuse and intentional discrimination (Fernández-Martínez \& Fernández, 2020), but also might further an information and power asymmetry between 
candidates and potential employers, leaving applicants with less room to negotiate (Sánchez-Monedero et al., 2020).

Overall, extant research has agreed that AI recruiting practices constitute a potential privacy loss for applicants attended by a greater power imbalance between applicants and employers; this poses an ethical risk. In addition, the use of more personal data, which may lead to more accurate predictions, is controversial (see also the next section). Thus, it is currently an unresolved normative question the extent to which a company may legally and ethically collect, store, and use personal data from applicants, such as the information available on social media platforms (Lin et al., 2020).

\section{Consistency, Accuracy and Validity}

There is broad agreement in extant literature that AI enables companies to make decisions more consistently across candidates and time (van den Broek et al., 2019). Whereas traditional assessment techniques such as analogue interviews are difficult to standardize, AI-based practices allow firms to put all applicants through exactly the same experience, resulting in an increase in the consistency of candidate assessment (Chamorro-Premuzic, 2019).

However, the accuracy and validity of the new AI assessment methods are controversially discussed. Today, employers do not necessarily know exactly which characteristics make an applicant a good fit for a given role. Studies have shown a very small correlation between a person's academic grades and their professional performance; still, many companies make above average grades a requirement for application. In contrast, some articles (e.g., Chamorro-Premuzic et al., 2019; Polli et al., 2019) argued that the new AI technologies have the potential to make the selection process more accurate as hiring algorithms predict a candidate's work-related behavior and performance potential based on the data of current top performers. AI may thereby outperform human inferences of personality in accuracy because it can process a much larger range of behavioral signals (Chamorro-Premuzic \& Akhtar, 2019; Chamorro-Premuzic et al., 2016; Polli et al., 2019). In this way, the use of AI improves both the possibilities of "what" and "how" skills and abilities are measured (Ryan \& Derous, 2019).

One article pointed to the accuracy-fairness trade-off in recruiting decisions and stated that $\mathrm{AI}$ technologies constitute the opportunity to overcome it (Chamorro-Premuzic et al., 2019). Historically, research has shown that traditional cognitive ability tests have led to discrimination of underrepresented groups, such as candidates with a lower socioeconomic status. Thus, to increase diversity and create an inclusive culture, companies have often de-emphasized cognitive tests in hiring (Chamorro-Premuzic et al., 2019). However, AI may overcome this fairness-accuracy tradeoff by deploying more dynamic and personalized scoring algorithms that can optimize for both (Chamorro-Premuzic et al., 2019; Raghavan et al., 2020).

Nevertheless, critics have raised concerns about the technical robustness and validity of AI-powered assessment methods. First, many of the newly offered AI tools have emerged as technological innovations, rather than from scientifically derived methods or research programs. Although there has been broad psychological research on the validity of traditional methods for candidate assessment, such as job interviews, assessment centers, or cognitive ability tests, the newly emerging AI tools have not been sufficiently scientifically validated, with regard to the underlying criteria for the prediction of job performance (Chamorro-Premuzic et al., 2016; Dattner et al., 2019; Raghavan et al., 2020). This means that firms may reject candidates based on unexplained correlations and make decisions based on factors with no clear causal connection to job performance (Cappelli, 2019; Kim, 2017). When AI links the tone of voice to differences in job performance, it raises the additional ethical question of whether it is appropriate to screen out people based on physically determined and rather unchangeable attributes (Dattner et al., 2019). Moreover, the indirect measurement of personality itself is still an open and discussed topic (De Cuyper et al., 2017).

Second, technical implementation bears some risks. For example, Tambe et al. (2019) argued that good employees are hard to measure, as it is difficult to disentangle individual from group performance. Further, introducing technological context, such as video games or avatar interviewers, to the recruiting process may add noisy variance to applicants' performance and, thus, measurement error (Ryan \& Derous, 2019). Therefore, a constant re-validation and control of the algorithmic tools is crucial. However, AI software vendors often do not publicly communicate whether or how they conduct validation studies on their models (Raghavan et al., 2020).

Third, Fernández-Martínez and Fernández (2020) brought up the risk that AI might not work equally for many people, undermining its accuracy. Along these lines, several studies (Buolamwini \& Gebru, 2018; Raji \& Buolamwini, 2019; Rhue, 2018) have shown that facial recognition software performs rather poorly, suffering from disparities in error rates across gender and race. Finally, Tambe et al. (2019) reported that AI recruiting faces the challenge of making trade-off decisions between accuracy and other ethical principles. For example, the authors stated that more "complicated" algorithms are more accurate, but they are also harder to explain, resulting in a trade-off between accuracy and explainability (we discuss the latter in the next paragraph).

These concerns about potential lack of validity and accuracy result in the question of whether it is ethical to use these new AI tools compared with more longstanding psychometric assessments that have been scientifically derived 
and validated. Which features are predictive, which are not, and which are protected? In particular, the selection of the features that define a good candidate is an ethically laden decision, about which current literature is ambivalent and further scientific validation is necessary (Schumann et al., 2020).

\section{Transparency and Explainability}

Another ethical opportunity mentioned in extant literature is the ability to establish transparency by providing applicants with updates and feedback throughout the process and in a timely fashion (e.g., via chatbots and AI technology), which can be considered one element of fair treatment (van Esch \& Black, 2019). Often, firms fall short of providing relevant information in a timely manner, or they provide no information other than confirmation that a candidate's application has been received. This can be very frustrating for candidates. However, next to progress updates, AI further enables firms to generate detailed feedback and give millions of job applicants data-driven insights on their strengths and development needs (Dattner et al., 2019).

However, the use of AI can also lead to a lack of transparency toward applicants, when the use of AI and automated systems is not proactively communicated to candidates (Sánchez-Monedero et al., 2020). Moreover, the predictive and decision-making processes of algorithms are often opaque, even for the programmers themselves. When algorithms take millions of data points for the assessment of a candidate, it becomes difficult to provide a qualitative explanation of which attributes are driving the decisions (Raghavan et al., 2020; Simbeck, 2019). This is ethically critical in the personnel selection context, due to its high relevance for people's lives, and because this kind of blackbox system may remain unchallenged, thereby obscuring discrimination (e.g., Tambe et al., 2019; Vasconcelos et al., 2018). Therefore, the GDPR also warrants a "right to explanation," by which people can ask for explanations about (algorithmic) decisions made about them (Pena et al., 2020).

Overall, the ethicality of AI recruiting depends highly on the mode in which it is implemented and used. On the one hand, it offers a huge ethical opportunity in the form of timely feedback for applicants; on the other hand, it bears the ethical risk of omitting transparency and explainability. Extant literature agrees that companies and recruiters should not rely on information produced by a black-box algorithm they do not fully understand. This is an open technical challenge to solve: building algorithms and $\mathrm{AI}$ applications that lead to explainable results (Schumann et al., 2020).

\section{Accountability}

Closely related to the issue of explainability is the topic of accountability in the hiring decision-making context. When automated AI technologies are used for decision-making, a question arises of whose job it is to adhere to ethical norms and labor laws, and who can be held responsible and accountable for the decisions made: the data scientists, the hiring managers or the company as a whole? This question becomes even more difficult when firms are not developing the AI themselves, but instead buying the technology from third-party vendors who want to protect their intellectual property and may not be willing to grant full transparency into the algorithms used (Sánchez-Monedero et al., 2020; Tambe et al., 2019). Lin et al. (2020) outlined that in the recruiting process, agents with different roles in the collective decision-making process can have a collective responsibility (i.e., each agent fulfills his or her role and shares a collective responsibility). Thus, when a recruiter makes a morally wrong decision based on a problematic recommendation by an AI, which in turn results from the negligence of a software engineer, both the recruiter and the engineer are collectively responsible and accountable for the wrong decision. Building on this discussion, Bornstein (2017) and Kim (2017) claimed that current regulation should be broadened, making companies that apply AI recruiting practices fully liable for any occurrence of discrimination or implicit bias in employment decisions.

It is clear that the AI itself cannot be held accountable; it should be a human agent who is ultimately responsible for the decision made when selecting an employee (Lin et al., 2020). However, the use of AI results in an obfuscation of responsibilities and accountabilities, which represents an ethical risk and must be clarified.

\section{Human Oversight and Autonomy}

The extent to which AI is integrated into the decision-making process varies across businesses. Some papers (Fernández-Martínez \& Fernández, 2020; Yarger et al., 2020) have reported that increasingly more tasks are taken over by algorithms, though firms still rely on human recruiters to make the final decision. However, other papers (e.g., Lee, 2018; Vasconcelos et al., 2018) have stated that AI has already taken over the automated decision-making process, forwarding or rejecting candidates. This raises the question of whether it is ethical to base hiring decisions solely on algorithms and without human intervention. Sánchez-Monedero et al. (2020) even raised the point of whether, due to the new GDPR regulation, it is in fact illegal to use a solely automated hiring system in the EU, because the GDPR grants people the right to a "human in the loop." Overall, extant literature agrees that the loss of human oversight should be 
avoided, but human involvement in the training, validation, and deployment process should be maintained.

Additionally, Lin et al. (2020) raised the question of whether the usage of AI effects human autonomy. When AI applications and analyses shape human decisions by interfering with deliberation processes, the violation of human autonomy can become a serious ethical concern. The authors called this "AI paternalism" (p. 16). However, this topic is not further discussed in the identified literature. Thus, questions regarding how AI impacts the autonomy and dignity of candidates remain open.

\section{Efficiency Gains and Effects on Internal Organization}

In the first place, AI-advanced selection tools are attractive for organizations, as they make hiring more cost- and timeefficient (e.g., Lee, 2018; van Esch \& Black, 2019). With the help of AI, employers have a greater ability to quickly shortlist candidates with high potential and streamline the selection process (Hipps, 2019; Persson, 2016; Savage \& Bales, 2017). For example, AI technology provides firms with the ability to initially screen and process hundreds of applications in a short time frame (Persson, 2016). Moreover, AIpowered video interviews increase efficiency by reducing selection process time as well as candidate time and travel distances (Fernández-Martínez \& Fernández, 2020).

However, the use of AI has further effects on the internal organization. The enhancement of recruiters' jobs is thereby considered an ethical opportunity of AI-enabled recruiting practices (Rąb-Kettler \& Lehnervp, 2019; van Esch \& Black, 2019). Daily, recruiters are confronted with numerous repetitive tasks, such as screening resumes, scheduling interviews and conducting similar conversations. When these tasks are taken over by AI, it results in a more meaningful job, as recruiters can undertake activities of higher value for the company. For instance, they can adapt better engagement techniques to ensure that a leading candidate accepts a job offer (Hipps, 2019; van Esch \& Black, 2019) and can better focus on the individual candidates, stepping from a pure head hunter role into a career guide role (Rąb-Kettler \& Lehnervp, 2019). Although the identified articles evaluated the effects of AI recruiting on the internal organizational members very positively, they must be studied in greater detail. For example, it needs to be tested whether a greater volume of candidates may prevent any gains in work time for recruiters (Ryan \& Derous, 2019). Further, potential job losses of recruiters are not yet part of the discussion.

\section{Perceived Fairness}

Although the research on applicant reactions to technologypowered recruiting processes has increased in recent years (see Woods et al., 2020 for a review on applicant reactions to digital selection procedures), there is limited understanding of how people perceive AI recruiting and contrasting findings exist. Several studies of applicant reactions to AI interviews provide some cause for concern as they reveal that applicants perceived AI interviews as less fair and less favorable than face-to-face interviews with humans (Acikgoz et al., 2020; Lee, 2018; Newman et al., 2020). For example, Lee (2018) found that participants believe that AI lacks certain human skills that are required in the recruiting context: It lacks human intuition, makes judgments based on keywords, ignores qualities that are hard to quantify and is not able to make exceptions. Furthermore, some participants felt that using algorithms and machines to assess humans is demeaning and dehumanizing (Lee, 2018). In contrast to those findings, another group of papers (Langer et al., 2019a, 2019b, 2020; Suen et al., 2019) found no differences in perceived fairness between interviews with an AI and interviews with a human among job applicants, although most of them exhibited lower favorability to AI interviews.

Other studies (Gelles et al., 2018; Kaibel et al., 2019; Langer et al., 2018; van Esch \& Black, 2019) examined the effect of different contextual factors on applicant reactions to the use of AI in hiring. For instance, Langer et al. (2018) found that applicants with a computer science background did not perceive AI recruiting differently from non-computer science applicants. Another study by Kaibel et al. (2019) examined the moderating effect of applicants' discrimination experience and uniqueness. They found that applicants who have experienced discrimination before perceive selection processes as fairer when an algorithm instead of a human makes the decision, whereas the negative effect of AI-based selection decisions on organizational attractiveness was stronger for individuals with a high sense of personal uniqueness. Underlining the relevance of perceived fairness, a study (van Esch \& Black, 2019) found that the more job candidates perceive the AI-enabled recruiting system as providing fair treatment, the likelier they are to engage in and complete the recruiting process.

In a case study, van den Broek et al. (2019) found that different stakeholder groups may hold different and clashing notions of fairness, which may even be reconsidered during the implementation of AI recruiting in practice. For example, although AI tools are introduced to make the process fairer and decisions consistent across the company, it was observed that some recruiters did not use the algorithmic results consistently, but made exceptions, which they perceived as fairer.

Overall, there is no clear answer to the question of how AI recruiting is perceived. What is perceived as fair in one context may be judged differently in another. Although we found several studies examining the fairness perceptions of applicants, the perspective of current employees and HR managers on AI recruiting tends to be neglected. This leaves 
open the question of the extent to which HR managers trust and accept AI recruiting.

\section{Approaches to Mitigate Ethical Risks}

As shown in the previous section, the new AI technologies pose new challenges to regulation and governments, especially as they are being applied in recruiting. Some approaches to mitigating the emerging ethical risks in the AI recruiting context are discussed in extant literature.

\section{Governmental Regulation}

In the identified literature, it has been broadly claimed that more governmental regulation is needed to respond to the new developments in hiring: Whereas Kim (2017) argued for a legal response to what she called classification bias, Fernández-Martínez and Fernández (2020) called for governments to track selection processes and check for any infringement of fundamental employment laws or human rights. In their recent analysis, Raghavan et al. (2020) found that currently, vendors' practices in bias mitigation are heterogeneous. This suggests that evolving industry norms are sensitive to bias concerns but lack clear guidance on how to respond. However, as current regulation leaves room for unethical behavior of firms, today, employers need to think beyond governmental law when developing and using predictive hiring tools (Bogen, 2019).

\section{Organizational Standards}

Extant literature refers to various organizational standards that firms may and should implement to ensure ethical use of AI in recruiting. First, it is suggested that companies applying AI tools in the personnel selection process comply with privacy laws just as they would in traditional hiring. On the one hand, this means that organizations should fully protect and keep safe all sensitive data. On the other hand, recruiters should not use or predict any private or sensitive candidate information in the recruiting process. In addition, firms should proactively and fully brief candidates that their data will be analyzed by AI systems and obtain their consent (e.g., Chamorro-Premuzic \& Akhtar, 2019; Simbeck, 2019). Second, firms should proactively and explicitly provide meaningful information on the hiring decision-making process, including information about the algorithmic techniques and data sets used, to ensure transparency and craft effective policy (Köchling et al., 2020; Raghavan et al., 2020; Sánchez-Monedero et al., 2020). Additionally, it should be always transparent to applicants whether they are communicating with another human or with AI (Simbeck, 2019). Third, several papers (e.g., Chamorro-Premuzic \& Akhtar, 2019; Köchling et al., 2020) also suggested human oversight on $A I$ as a standard for organizations. The authors encouraged a human review, in which experienced recruiters oversee the selection and evaluation made by AI. They argued that decisions should be made by an algorithm-informed human, rather than by an algorithm alone. Fourth, to further ensure and audit the implementation of these ethical standards, various authors have referred to compliance instruments companies should establish, such as an AI ethics board with an oversight function, consisting of representatives of relevant stakeholders who debate the data and ethical dimensions of $\mathrm{AI}$ algorithms and agree on boundaries for AI technology in the company (Simbeck, 2019; Tambe et al., 2019). In addition, Tambe et al. (2019) recommended specifying a code of ethics for AI-related initiatives within the company. Lastly, authors have encouraged diverse data scientist teams in organizations to foster inclusion and equity in AI (Giang, 2018; Yarger et al., 2020). In particular, in the ML algorithm development process, diverse voices across gender and race must be present to raise questions and check implicit assumptions.

\section{Technical Due Diligence}

Next to approaches on the governmental and organizational level, the identified literature also discusses technical methods to ensure ethical application of AI tools in recruiting. First, authors mentioned the data literacy of programmers, as well as the knowledge of hiring managers on how to use the AI solutions as a first prerequisite. Given that any data concerns can have a life-changing impact on applicants, companies need to have adequate levels of data and statistical skills to assure the accuracy and validity of the developed algorithms (Fernández-Martínez \& Fernández, 2020; Lewis, 2018; Simbeck, 2019). Second, if companies do not develop the algorithms in-house, but buy more innovative skill tests or games from external vendors, practitioners are strongly encouraged to refer to professional test standards and obtain critical information about the tools: for example, evidence that informs psychometric reliability, criterion-related validity and bias implications (Oswald et al., 2020).

Third, the ethicality of the AI tool design, which should include bias mitigation techniques, plays a crucial role. For instance, some AI software vendors remove any wording or phrases that can unconsciously predict the gender of a candidate from $\mathrm{CV}$ s to circumvent unconscious bias and improve equity (e.g., Lin et al., 2020; Yarger et al., 2020). A different approach suggested by Williams et al. (2018) is to proactively gather and use social category data to illuminate and combat discriminatory practices. The authors argued that only when data are labeled with social categories can data scientists detect, understand, or remediate patterns of discrimination. Furthermore, open-source tools and technical frameworks for data scientists (e.g., IBM's "AI Fairness 
360") can facilitate systematic bias checks and assist developers in embedding fairness in their algorithms (see Mujtaba \& Mahapatra, 2019 for an overview of open-source toolkits). However, Sánchez-Monedero et al. (2020) pointed to the computational limitations of bias mitigation techniques and further argued that most bias mitigation systems aim at meeting the constraints of US law, which makes them not directly applicable in EU markets. In the context of ethical AI, Polli (2019) further referred to the movement among AI practitioners to develop a set of design principles for making AI ethical and fair (i.e., beneficial to everyone). She thereby emphasized the key principle according to which AI should be designed so that it can be easily audited. Rather than just assuming that algorithms yield accurate results, employers must regularly check the technology used for discrimination, as well as data errors and biases (e.g., Fernández-Martínez \& Fernández, 2020; Hipps, 2019; Polli, 2019). Efforts must be made to constantly improve the robustness of any AI tool and, thus, proactive auditing methods should be implemented (Köchling et al., 2020). For example, outside professionals can be hired to build an internal auditing team to look at the AI decisions and audit key algorithms (Giang, 2018; Mann \& O'Neil, 2016). They can carry out random spot checks on algorithmic recommendations, investigating in detail which candidates the algorithm has been selecting and why. To this end, Fernández-Martínez and Fernández (2020) developed an automated multi-agent software architecture to support auditing the recruiting process.

Lastly, companies need to be able to explain why a candidate has been selected and the causality regarding which specific attributes can be associated with their success in a role (Chamorro-Premuzic et al., 2019; Lewis, 2018). Thus, employers should not rely on black-box models, but develop AI applications that are interpretable (Lin et al., 2020). Transparency on algorithmic assumptions and models (e.g., in the form of explainability reports) is key in the mitigation of bias and when addressing trade-off decisions data scientists have to make (e.g., Mujtaba \& Mahapatra, 2019; Tambe et al., 2019).

\section{Awareness Among Employees}

AI plays a critical role in technology to attack the diversity problem. It is therefore crucial that companies invest not only in AI technology, but also in people who are aware of both the opportunities and the risks that attend AI-powered recruiting practices (Chamorro-Premuzic et al., 2019). The awareness and sensibility of recruiters and data scientists about the potential bias and shortcomings of their algorithms is key to address the accompanying ethical challenges (Simbeck, 2019). When regulation is not enough to guide human behavior, ethical thinking and awareness of conscious use of predictive AI tools must be further promoted beyond regulation (Persson, 2016).

\section{Discussion}

Overall, we make four observations from structuring and synthesizing the current literature. First, this review indicates that there are various streams addressing ethical considerations of AI-based recruiting, but that insufficient attention has been given to ethical theories in this context. As various extant articles have a practitioner, legal, technical or descriptive focus, they tend to mention ethical considerations, but avoid normatively assessing them from a theoretical perspective. We identified only three theoretical articles, underlining the lack of a theoretical foundation within this field of research to date. However, by exploring the ethicality of AI recruiting, additional work based on ethical theory could prove beneficial to managers and organizations. Our review has shown that ethicality underlies the law and regulations in this area, but goes beyond them as well. Thus, more theoretical and normative papers are needed to provide organizations with a set of perspectives and suggested actions that may be taken to enhance morality in hiring (Alder \& Gilbert, 2006).

Second, we found that some ethical concerns are prevalent in extant research, whereas others have not been sufficiently discussed. Most articles focus on human and algorithmic bias, whereas, for example, critical thoughts about accountability for AI-based recruiting practices, which were only mentioned in five of the 51 papers reviewed, are underrepresented. Thus, the field lacks an explicit discussion of the accountability of organizations for AI applications in recruiting, although this is a fundamental concern of today's research on ethics of AI, which treats the responsibility gap that may arise when an AI technology makes decisions independently and without direct human control (Johnson, 2015; Martin, 2018; Mittelstadt et al., 2016). Similarly, AI's impact on human autonomy and dignity, which is often considered an important principle for ethical AI (e.g., Floridi et al., 2018; University of Montreal, 2018), has only been briefly mentioned by one article and has not been assessed in detail.

Third, the identified solution approaches to mitigating ethical risks of $\mathrm{AI}$ applications are rather general and not specifically tailored to the recruiting context. They resemble the recommendations given in extant AI ethics guidelines. For instance, some of the mentioned solution approaches can be similarly found in the methods proposed by the High-Level Expert Group (High-Level Expert Group on Artificial Intelligence, 2019) to help implement trustworthy AI. However, a domain-specific focus would be desirable, because general normative guidelines do not have a tangible 
impact in many cases, precisely because of their generality and superficiality (Hagendorff, 2020). Instead, concrete implementation guidelines should be sensitive to contextual details and speak to the domain-specific regulation (HighLevel Expert Group on Artificial Intelligence, 2019). In addition, ethical guidelines would benefit from being supplemented with detailed technical explanations. This would bridge the gap between abstract ethical principles and concrete technological implementations, for example by defining what it really means to implement privacy or transparency in AI systems in a given context (Hagendorff, 2020).

Finally, our systematic review reveals a predominance of non-empirical work. Only 18 articles (35\% of papers reviewed) are empirical. Most examine perceived fairness and provide contrasting findings. Therefore, the ways in which people react to decisions made by $\mathrm{AI}$ in the recruiting context are still not well understood. Additional empirical research in this area is desirable, because respective findings may guide organizations on how to best use AI in selection to attract and retain top talent. Our mapping of ethical considerations reveals that there are other research topics, besides perceived fairness, that remain ambiguous. Topics such as the use of personal data or AI's impact on workforce diversity and assessment validity could also benefit from empirical evaluation.

In line with the findings of Chamorro-Premuzic et al. (2016), we observed that academic research struggles to keep pace with the rapidly evolving technology, allowing firms and vendors of recruiting technology to push the boundaries of justifiable selection practices. Addressing the identified research gaps, the following sections provide a more detailed roadmap for theoretical and empirical directions to advance research as well as a discussion of the practical implications of our findings.

\section{Implications for Future Theoretical Research}

Our review shows that insufficient attention has been given to relevant ethical frameworks in the research on the ethicality of AI-enabled recruiting. In fact, we did not find any article that provided a normative ethical analysis of AI recruiting by linking it to an established ethical lens such as a utilitarian, deontological or contract theory perspective (see Table 3). Thus, the emerging topic would benefit from being assessed through the lens of ethics theory, showing how these ethical schools would characterize morally relevant aspects of AI recruiting.

For example, one topic mentioned in our review that may be discussed controversially across the traditional schools is the ambit of privacy. As scraping social media platforms or face recognition techniques can gather and assess highly personal information on applicants' personality, health status, or sexual orientation, AI recruiting tools can be considered quite invasive technologies. On the one hand, contractarians, who pride themselves on their defense of private freedom from outward intrusion should find this problematic. Deontologists, who find morality in adherence to universal obligations, might agree with them in this assessment, especially when the respective information is gathered via an untransparent process. On the other hand, utilitarians, who see the greatest good or happiness for the greatest number of people as the most important value, are skeptical toward a strict differentiation between "the private" and "the public" (Seele et al., 2019). Thus, they might prefer to base their assessment on the practical consequences of employing applicant profiling based on private data. If this practice leads to the outcome that each position is filled by the best candidate, then the greatest good for the greatest number will usually be accomplished (Alder \& Gilbert, 2006): The company will benefit from high productivity and enhanced competitiveness, customers and society will benefit from better products and services, shareholders will benefit from increasing profits, and also the employees will benefit from a higher job satisfaction.

A similar argumentation may apply to the topic of transparency and explainability. The implementation of transparent processes and explainable decision-making may not be important for utilitarians as long as the best candidates are hired. However, the deontological view may argue that the greatest good for the greatest number does not justify violating individuals' rights. Whereas, at this point, we have exemplified possible lines of argumentation of the classical ethical schools, future research should comprehensively delve deeper into each of the identified topics in our review and draw on major streams of ethical thinking, to mark and classify instances where AI recruiting would be approved or rejected by that ethical school.

Furthermore, AI recruiting should reconnect to the applied ethics fields of business ethics and AI ethics, where stronger theoretical contributions may be generated. Within business ethics research, the social contracts theory (Donaldson \& Dunfee, 1994, 1999, 2000) perspective might be worth employing. Martin (2016) applied this approach to technology and online privacy, recognizing that people develop micro-social contracts with each provider, technological artifact, and circumstances as they navigate the increasingly interconnected world. By building on and extending Nissenbaum's framework of contextual integrity (2004, 2009), Martin (2016) further argued that stakeholder complaints about privacy violations are often due to changes in social contracts without consultation and approval. Future research could draw from this work to advance the field of AI recruiting using this micro-social contract narrative as a theoretical construct. This requires a detailed examination of expectations about ethical standards in the recruiting process, such as privacy and transparency, from an applicant 
perspective, as well as an analysis of whether, in this context, new technologies come into conflict with underlying norms.

Moreover, the field of AI recruiting and selection ethics can be positioned in the broader ongoing discourse on AI ethics. Current advances in the development and application of AI have, in recent years, been accompanied by the release of several ethics guidelines by various stakeholder groups. These include, for instance, the Montreal Declaration for Responsible AI (University of Montreal, 2018), the Ethics Guidelines for Trustworthy AI of the High-Level Expert Group on AI set up by the European Commission (HighLevel Expert Group on Artificial Intelligence, 2019), and the AI4People's principles for AI ethics (Floridi et al., 2018; see Fjeld et al., 2020; Hagendorff, 2020; or Jobin et al., 2019 for a meta-analysis). In these documents, normative principles are developed to harness the disruptive potential and to tackle potential cases of misuse of AI technologies. Although these guidelines offer high-level guidance for AI applications in general, they need to be further tailored to the domain-specific use cases of AI, such as the recruiting context (High-Level Expert Group on Artificial Intelligence, 2019). What is deemed an appropriate action may depend on the domain in which AI is used and may differ between recruiting and other domains. To this end, there is a strong need for domain-specific works (Tolmeijer et al., 2020).

Thus, future research could build upon the general AI ethics frameworks and derive detailed guidelines for their operationalization in the recruiting context. For instance, concrete guidelines could be built on the AI4People's work (Floridi et al., 2018), which first proposed five principles to guide AI ethics: beneficence, non-maleficence, autonomy, justice, and explicability. Within each principle, concrete guidelines and ethical questions related to AI recruiting could be outlined. Thereby, the input from domain experts would be as important as the input from AI developers, implying the need for close collaboration between disciplines. Computer scientists and philosophers as well as domain experts and social science experts would have to work together to ensure the desired effects of ethical AI (Tolmeijer et al., 2020). Drawing from AI ethics frameworks could inform a more holistic view of ethical considerations in AI-enabled recruiting practices. Moreover, detractors' critiques that $\mathrm{AI}$ ethics initiatives provide few practical recommendations because they are vague and high level (Hagendorff, 2020; Mittelstadt, 2019) would thereby be rebutted.

\section{Implications for Future Empirical Research}

In summary, our review revealed several ethically ambivalent topics related to AI recruiting, which should be addressed by future empirical research. First, future research needs to better understand the accuracy and validity of AI recruiting tools (Woods et al., 2020). In this context, relevant questions are, for example: What are the criterion validities of different forms of AI in recruiting? Does AI recruiting outperform traditional selection procedures in terms of validity in any specific situations? To answer these questions, it may not be enough to establish measurement equivalence with traditional methods, which has been undertaken in the past, for example, when evaluating web-based assessment tools (e.g., Ployhart et al., 2003). Instead, research needs to approach the validation of AI assessment tools in their own right, rather than benchmarking it against traditional formats (Woods et al., 2020). To this end, quantitative studies that examine the validity of AI tool predictions, for example based on some measures of job performance, should be conducted. This has also been a common research design in the field of industrial, work and organizational (IWO) psychology for the examination of traditional recruiting methods (see, for example, Aguado et al., 2019).

Second, in our review, we touched on critical concerns related to informed consent and the use of personal data, which could be explored empirically in greater detail. In line with North-Samardzic (2019), we propose that future research could build on the findings of Hoofnagle et al. (2010) and Park (2013) by examining whether candidates are sufficiently informed about how AI is used during their application process and whether they understand the implications of AI technologies to be able to consent properly. Research is needed to clarify fundamental questions about the factors that determine applicants' privacy concerns. There may be differences between countries and cultures, attributable to differences in cultural and contextual factors, as well as privacy and data protection laws. A quantitative research design, e.g., in the form of online surveys, may be a suitable research design in this context (see, for example, Jeske \& Shultz, 2019). The implications of research on these issues would help hiring managers shape their recruiting process and improve related privacy policies, ensuring an effective recruiting procedure (Woods et al., 2020).

Third, empirical studies on AI's effect on workforce diversity would be highly beneficial for the ongoing debate. This kind of empirical evidence would finally determine whether algorithms have the potential to overcome bias in hiring to establish diverse workforces. To this end, experimental research designs that examine the differences in decisions made by recruiters compared to AI decisions may be applicable. In addition, field data will be needed to increase external validity and make a final judgment on whether AIbased recruiting represents this huge ethical opportunity of more diverse workforces.

Finally, future research should further investigate and better understand the perceived fairness of AI recruiting. Adverse applicant reactions could have severe impacts for firms, as they might lead to negative outcomes, such as public complaints. Thus, applicant reaction research can 
offer relevant and practical advice for system designers and recruiters (Yarger et al., 2020). People's attitudes toward technologies have changed throughout history, and the same is expected to happen with the perception of AI applications, including AI-based recruiting tools (Lee, 2018). Thus, it would be interesting to study people's attitudes and perceptions of fairness over time, while increasingly more companies deploy AI tools in their recruiting processes and develop a current and up-to-date view on applicant reactions. Future research should thereby further shed light on the contextual and interactional factors that influence people's perception of AI-based recruiting decisions, because ethical concerns are often related to context (North-Samardzic, 2019; van den Broek et al., 2019). For instance, the role of the degree of an applicant's interaction with the AI could be examined (Lee, 2018). Applicants who directly interact with AI (e.g., via a chatbot or a video interview with a virtual AI agent) might perceive the AI-based procedure differently from applicants who do not interact with the AI, but whose $\mathrm{CVs}$ and test results have been analyzed by AI. Furthermore, the design features of gamified AI assessments (e.g., easeof-use, mobile hosting or the nature of games themselves) and the positioning of AI tools in different stages of the hiring process could similarly affect reactions (Woods et al., 2020). Moreover, the type of job, the industry context, the cultural background, and other individual or demographic differences might affect an applicant's perception and are worth studying in greater detail.

While studying applicant reactions, it seems appropriate to primarily use a survey experiment methodology based on hypothetical situations or, alternatively, a lab design, in this early stage of research in this area (Woods et al., 2020). Nevertheless, these must be complemented with field studies involving people's actual experiences in high-stake selection situations to increase the external validity and generalizability of the findings (Acikgoz et al., 2020; Lee, 2018). Future research could thereby benefit from building upon Gilliland's (1993) theories of organizational justice, which explain factors that affect the perceived fairness of a selection system. However, it needs to go beyond that to re-define the changing nature of procedural justice in the context of AI recruiting, as well as the associated impacting factors and outcomes (Woods et al., 2020). Alternative models from the field of technology acceptance, such as Davis's (1989) technology acceptance model (TAM) or Venkatesh et al.'s (2003) unified theory of acceptance and use of technology (UTAUT), may also contribute to a better understanding of reactions to new technology in selection (Brenner et al., 2016). These models identify the core determinants of people's acceptance of new technologies, which may also be good predictors of applicants' reactions to the use of AI in recruiting.

\section{Implications for Practice}

By synthesizing and evaluating the ethical considerations around AI recruiting in the extant literature, our review provides implications for practice. We identified the core opportunities and risks of $\mathrm{AI}$-enabled recruiting and selection practices, as well as a set of practical approaches to mitigate the latter. On the one hand, our review shows the ethical opportunities AI offers, such as the reduction of human bias in hiring or the ability to give timely and detailed feedback to applicants, which could help managers attain greater legitimacy within their organizations, as well as society, for their recruitment practices. On the other hand, our work stresses the importance of companies being aware of ethical risks that accompany the implementation of $\mathrm{AI}$ in recruiting. Even if AI software vendors advertise the avoidance of human bias, algorithms may be biased due to technical shortcomings, such as biased training sets or algorithmic design. Problems become even more complex when algorithms are based on ML and develop individually, so that developers are no longer able to explain how the AI has come to its decisions. Moreover, companies should be aware that the validity of the decisions made is not only determined by the AI itself, but also the underlying criteria used to predict job performance, which may not be scientifically validated (Chamorro-Premuzic et al., 2016; Dattner et al., 2019; Raghavan et al., 2020).

Overall, we observed contrasting views in the identified literature on the ethicality of AI recruiting. Even if we cannot offer a conclusive evaluation of whether the ethical opportunities outweigh the risks, managers need to understand the ethical concerns AI technologies might create and that algorithmic decisions might contradict what they aim to do with their workforce (Hickok, 2020). Thus, they must consider approaches to address those ethical concerns. In our review, we provide an overview of such practical approaches mentioned in the identified literature, although this list does not claim to be exhaustive.

As governmental regulation currently leaves room for unethical behavior of companies, firms should think and act beyond regulation and establish organizational standards to ensure the ethical use of AI recruiting tools. These might include compliance with privacy laws, transparency on AI usage, and human oversight on the AI in place. In addition, organizational compliance mechanisms, such as AI ethics boards or a code of ethics, could help to ensure ethical use of AI within firms. Indeed, in his study, Somers (2001) found that the presence of a corporate code of ethics is associated with less perceived wrongdoing in organizations. However, the author also pointed out that formal ethics codes should be considered as "one component of a milieu that encourages and supports high standards of ethical behavior" (p. 
194) and that such codes need to be reinforced by supportive measures and values. Thus, to mitigate the ethical risks of AI applications in practice, a multi-tier approach is needed that includes all kinds of measures mentioned in our review, covering organizational standards, as well as technical due diligence and awareness among employees. It is crucial to anchor ethics competencies at the team and individual levels within organizations, e.g., via the implementation of diverse data scientist teams. Given that manifold ethical questions may arise in the development of algorithms, diverse voices and people who are aware of the potential shortcomings of recruiting algorithms are needed to check implicit assumptions and foster inclusion and equity.

Only by proactively tackling the ethical concerns, both in implementation and in external communication, can practitioners create new forms of AI recruiting practices that are both efficient and effective, and which also have the potential to manifest a competitive advantage and financial payoff (Bartneck et al., 2021).

\section{Conclusion}

AI tools have already become part of today's recruiting and selection practices. Our review of the literature on ethical consideration of AI-enabled recruiting organizes the extant research, which is still in an emerging stage. The topic is addressed from theoretical, practitioner, legal, technical and descriptive perspectives. By synthesizing the identified articles and ethically evaluating the considerations made, we provide researchers with guidance on the current state of the literature and establish a common basis for future research in the field. Furthermore, we identify gaps in extant research and reveal future research opportunities. A need exists for theoretical and empirical research bridging the gap between business ethics and AI recruiting applications in practice. Because the development and deployment of AI recruiting practices are increasing and come with a variety of ethical risks and ambiguities, we hope that our review will stimulate research to address the many remaining unstudied areas of AI-enabled recruiting and selection.

\section{Notes}

1. Grouping the 51 articles into the five categories yielded an average agreement of $86 \%$. Cohen's kappa ( $\kappa)$ was 0.815 between the two raters, indicating "almost perfect" agreement (Landis \& Koch, 1977, p. 165).

2. Disparate treatment discrimination refers to intentional discrimination based on protected attributes, where the employer intentionally treats people of a class protected under Title VII less variably than others.
3. Disparate impact or adverse impact discrimination refers to employment practices that appear neutral but have a discriminatory effect on a class protected under Title VII. The rule of thumb is the four-fifths rule: The selection rate for a protected group should not be less than four-fifths of the group with the highest selection rate.

Funding Open Access funding enabled and organized by Projekt DEAL.

\section{Declarations}

Conflict of interest The authors declare they have no conflict of interest.

Ethical Approval This article does not contain studies with human participants performed by the authors.

Open Access This article is licensed under a Creative Commons Attribution 4.0 International License, which permits use, sharing, adaptation, distribution and reproduction in any medium or format, as long as you give appropriate credit to the original author(s) and the source, provide a link to the Creative Commons licence, and indicate if changes were made. The images or other third party material in this article are included in the article's Creative Commons licence, unless indicated otherwise in a credit line to the material. If material is not included in the article's Creative Commons licence and your intended use is not permitted by statutory regulation or exceeds the permitted use, you will need to obtain permission directly from the copyright holder. To view a copy of this licence, visit http://creativecommons.org/licenses/by/4.0/.

\section{References}

Acikgoz, Y., Davison, K. H., Compagnone, M., \& Laske, M. (2020). Justice perceptions of artificial intelligence in selection. International Journal of Selection and Assessment, 28, 399-416. https:// doi.org/10.1111/ijsa.12306

Aguado, D., Andrés, J. C., García-Izquierdo, A. L., \& Rodríguez, J. (2019). LinkedIn "big four": Job performance validation in the ICT Sector. Revista De Psicología Del Trabajo y De Las Organizaciones, 35, 53-64. https://doi.org/10.5093/jwop2019a7

Alder, G. S., \& Gilbert, J. (2006). Achieving ethics and fairness in hiring: Going beyond the law. Journal of Business Ethics, 68, 449-464. https://doi.org/10.1007/s10551-006-9039-z

Barocas, S., \& Selbst, A. D. (2016). Big data's disparate impact. California Law Review, 104, 671-732. https://doi.org/10.15779/ Z38BG31

Bartneck, C., Luetge, C., Wagner, A., \& Welsh, S. (2021). An introduction to ethics in robotics and AI. Berlin: Springer.

Bauer, T. N., Truxillo, D. M., Tucker, J. S., Weathers, V., Bertolino, M., Erdogan, B., et al. (2006). Selection in the information age: The impact of privacy concerns and computer experience on applicant reactions. Journal of Management, 32, 601-621. https://doi. org/10.1177/0149206306289829

Bîgu, D., \& Cernea, M.-V. (2019). Algorithmic bias in current hiring practices: An ethical examination. In 13th international 
management conference (IMC) on management strategies for hiph Performance, Bucharest, Romania, October 31-November 1.

Blacksmith, N., Willford, J. C., \& Behrend, T. S. (2016). Technology in the employment interview: A meta-analysis and future research agenda. Personnel Assessment and Decisions, 2, 12-20.

Bogen, M. (2019). All the ways hiring algorithms can introduce bias. Harvard Business Review, May 6.

Bornstein, S. (2017). Reckless discrimination. California Law Review, 105, 1055-1110. https://doi.org/10.15779/Z388P5V86M

Brenner, F. S., Ortner, T. M., \& Fay, D. (2016). Asynchronous video interviewing as a new technology in personnel selection: The applicant's point of view. Frontiers in Psychology. https://doi. org/10.3389/fpsyg.2016.00863

Buolamwini, J., \& Gebru, T. (2018). Gender shades: Intersectional accuracy disparities in commercial gender classification. In S. Friedler \& C. Wilson (Eds.), Conference on fairness, accountability, and transparency (Vol. 81, pp. 1-15). ACM.

Cappelli, P. (2019). Data science can't fix hiring (yet). Harvard Business Review, May-June.

Chamorro-Premuzic, T. (2019). Will AI reduce gender bias in hiring? Harvard Business Review, June 10.

Chamorro-Premuzic, T., \& Akhtar, R. (2019). Should companies use AI to assess job candidates? Harvard Business Review, May 17.

Chamorro-Premuzic, T., Akhtar, R., Winsborough, D., \& Sherman, R. A. (2017). The datafication of talent: How technology is advancing the science of human potential at work. Current Opinion in Behavioral Sciences, 18, 13-16. https://doi.org/10.1016/j.cobeha. 2017.04.007

Chamorro-Premuzic, T., Polli, F., \& Dattner, B. (2019). Building ethical AI for talent management. Harvard Business Review, November 21 .

Chamorro-Premuzic, T., Winsborough, D., Sherman, R., \& A., \& Hogan, R. (2016). New talent signals: Shiny new objects or a brave new world? Industrial and Organizational Psychology, 9(3), 621-640.

Chapman, D. S., Uggerslev, K. L., \& Webster, J. (2003). Applicant reactions to face-to-face and technology-mediated interviews: A field investigation. The Journal of Applied Psychology, 88, 944-953. https://doi.org/10.1037/0021-9010.88.5.944

Chwastek, R. (2017). Cognitive systems in human resources. In Y. Demazeau, J. Gao, G. Xu, J. Kozlak, K. Muller, I. Razzak, et al. (Eds.), 4th international conference on behavioral, economic advance in behavioral, economic, sociocultural computing, Krakow, Poland, October 16-18, 2017. New York: IEEE.

Corbett-Davies, S., Pierson, E., Feller, A., Goel, S., \& Huq, A. (2017). Algorithmic decision making and the cost of fairness. In S. Matwin, S. Yu, \& F. Farooq (Eds.), International conference on knowledge discovery and data mining, Halifax, NS, Canada, August 13-17 (pp. 797-806). New York: ACM. https://doi.org/ 10.1145/3097983.3098095.

Dattner, B., Chamorro-Premuzic, T., Buchband, R., \& Schettler, L. (2019). The legal and ethical implications of using AI in hiring. Harvard Business Review, April 25.

Davis, F. D. (1989). Perceived usefulness, perceived ease of use, and user acceptance of information technology. MIS Quarterly, 13, 319-340. https://doi.org/10.2307/249008

De Cuyper, J., De Houwer, J., Vansteelandt, K., Perugini, M., Pieters, G., Claes, L., et al. (2017). Using indirect measurement tasks to assess the self-concept of personality: A systematic review and meta-analyses. European Journal of Personality, 31, 8-41. https://doi.org/10.1002/per.2092

Donaldson, T., \& Dunfee, T. W. (1994). Toward a unified conception of business ethics: Integrative social contracts theory. Academy of Management Review, 19(2), 252-284.

Donaldson, T., \& Dunfee, T. W. (1999). Ties that bind: A social contracts approach to business ethics. Harvard Business Press.
Donaldson, T., \& Dunfee, T. W. (2000). Precis for ties that bind. Business and Society Review, 105, 436-443. https://doi.org/10.1111/ 0045-3609.00092

Dwork, C., Hardt, M., Pitassi, T., \& Reingold, O. (2012). Fairness through awareness. In $3 r d$ conference on innovations in theoretical computer science, Cambridge, MA, USA, January 8-10 (pp. 214-226).

Fernández-Martínez, C., \& Fernández, A. (2020). AI and recruiting software: Ethical and legal implications. Paladyn: Journal of Behavioral Robotics, 11, 199-216. https://doi.org/10.1515/ pjbr-2020-0030

Fjeld, J., Achten, N., Hilligoss, H., Nagy, A. C., \& Srikumar, M. (2020). Principled artificial intelligence: Mapping consensus in ethical and rights-based approaches to principles for AI. Berkman Klein Center for Internet \& Society.

Florentine, S. (2016, December 22). How artificial intelligence can eliminate bias in hiring. CIO. . Retrieved Jul 20, 2020 from https://www.cio.com/article/3152798/how-artificial-intelligen ce-can-eliminate-bias-in-hiring.html.

Floridi, L., Cowls, J., Beltrametti, M., Chatila, R., Chazerand, P., Dignum, V., et al. (2018). AI4People-An ethical framework for a good AI society: Opportunities, risks, principles, and recommendations. Minds and Machines, 28, 689-707. https://doi.org/ 10.1007/s11023-018-9482-5

Gelles, R., McElfresh, D., \& Mittu, A. (2018). Project report: Perceptions of AI in hiring. University of Maryland. Retrieved Dec 18, 2020 from https://anjali.mittudev.com/content/Fairness_in_ AI.pdf.

Giang, V. (2018, May 8). The potential hidden bias in automated hiring systems. Fast Company. . Retrieved Aug 13, 2020 from https:// www.fastcompany.com/40566971/the-potential-hidden-bias-inautomated-hiring-systems.

Hagendorff, T. (2020). The ethics of AI ethics: An evaluation of guidelines. Minds and Machines, 30, 99-120. https://doi.org/10.1007/ s11023-020-09517-8

Hardt, M., Price, E., \& Srebro, N. (2016). Equality of opportunity in supervised learning. In 30th conference on neural information processing systems (NIPS), Barcelona, Spain, Dec 4-9 .

Hickok, M. (2020). Why was your job application rejected: Bias in recruitment algorithms? (Part 1). Retrieved Aug 13, 2020 from https://montrealethics.ai/why-was-your-job-application-rejectedbias-in-recruitment-algorithms-part-1/.

High-Level Expert Group on Artificial Intelligence. (2019). Ethics guidelines for trustworthy AI. Retrieved Aug 13, 2020 from https://ec.europa.eu/digital-single-market/en/news/ethics-guide lines-trustworthy-ai.

Hipps, C. (2019). Can AI reduce bias in talent selection? Training Journal, December, 22-24.

Hoofnagle, C. J., King, J., Li, S., \& Turow, J. (2010). How different are young adults from older adults when it comes to information privacy attitudes \& policies? Retrieved Aug 13, 2020 from http:// repository.upenn.edu/ascpapers/399.

Jayaratne, M., \& Jayatilleke, B. (2020). Predicting personality using answers to open-ended interview questions. IEEE Access, 8, 115345-115355. https://doi.org/10.1109/ACCESS.2020.30040 02

Jeske, D., \& Shultz, K. S. (2019). Social media screening and content effects: Implications for job applicant reactions. International Journal of Manpower, 40, 73-86. https://doi.org/10.1108/ IJM-06-2017-0138

Jobin, A., Ienca, M., \& Vayena, E. (2019). The global landscape of AI ethics guidelines. Nature Machine Intelligence, 1, 389-399. https://doi.org/10.1038/s42256-019-0088-2

Johnson, D. G. (2015). Technology with no human responsibility? Journal of Business Ethics, 127, 707-715. https://doi.org/10. 1007/s10551-014-2180-1 
Kaibel, C., Koch-Bayram, I., Biemann, T., \& Mühlenbock, M. (2019). Applicant perceptions of hiring algorithms - Uniqueness and discrimination experiences as moderators. Academy of Management Proceedings. https://doi.org/10.5465/AMBPP.2019.210

Kaplan, A., \& Haenlein, M. (2019). Siri, Siri, in my hand: Who's the fairest in the land? On the interpretations, illustrations, and implications of artificial intelligence. Business Horizons, 62, 15-25. https://doi.org/10.1016/j.bushor.2018.08.004

Kim, P. T. (2017). Data-driven discrimination at work. William \& Mary Law Review, 58(3), 857-937.

Kim, P. T., \& Scott, S. (2018). Discrimination in online employment recruiting. Saint Louis University Law Journal, 63, 93.

Kluemper, D. H., Rosen, P. A., \& Mossholder, K. W. (2012). Social networking websites, personality ratings, and the organizational context: More than meets the eye? Journal of Applied Social Psychology, 42, 1143-1172. https://doi.org/10.1111/j.1559-1816. 2011.00881.x

Köchling, A., Riazy, S., Wehner, M. C., \& Simbeck, K. (2020). Highly accurate, but still discriminatory: A fairness evaluation of algorithmic video analysis in the recruitment context. Business \& Information Systems Engineering. https://doi.org/10.1007/ s12599-020-00673-w

Konradt, U., Warszta, T., \& Ellwart, T. (2013). Fairness perceptions in web-based selection: Impact on applicants' pursuit intentions, recommendation intentions, and intentions to reapply. International Journal of Selection and Assessment, 21(2), 155-169.

Landis, J. R., \& Koch, G. G. (1977). The measurement of observer agreement for categorical data. Biometrics, 33(1), 159-174.

Langer, M., König, C. J., \& Fitili, A. (2018). Information as a doubleedged sword: The role of computer experience and information on applicant reactions towards novel technologies for personnel selection. Computers in Human Behavior, 81, 19-30. https://doi. org/10.1016/j.chb.2017.11.036

Langer, M., König, C. J., \& Hemsing, V. (2020). Is anybody listening? The impact of automatically evaluated job interviews on impression management and applicant reactions. Journal of Managerial Psychology, 35, 271-284. https://doi.org/10.1108/ JMP-03-2019-0156

Langer, M., König, C. J., \& Papathanasiou, M. (2019a). Highly automated job interviews: Acceptance under the influence of stakes. International Journal of Selection and Assessment, 27, 217-234. https://doi.org/10.1111/ijsa.12246

Langer, M., König, C. J., Sanchez, D.R.-P., \& Samadi, S. (2019b). Highly automated interviews: Applicant reactions and the organizational context. Journal of Managerial Psychology, 35, 301-314. https://doi.org/10.1108/JMP-09-2018-0402

Lee, M. K. (2018). Understanding perception of algorithmic decisions: Fairness, trust, and emotion in response to algorithmic management. Big Data \& Society. https://doi.org/10.1177/2053951718 756684

Leicht-Deobald, U., Busch, T., Schank, C., Weibel, A., Schafheitle, S., Wildhaber, I., et al. (2019). The challenges of algorithmbased HR decision-making for personal integrity. Journal of Business Ethics, 160, 377-392. https://doi.org/10.1007/ s10551-019-04204-w

Lepri, B., Oliver, N., Letouzé, E., Pentland, A., \& Vinck, P. (2018). Fair, transparent, and accountable algorithmic decision-making processes. Philosophy \& Technology, 31(4), 611-627.

Lewis, N. (2018, November 12). Will AI remove hiring bias? Strategic HR Review. Retrieved July 20, 2020 from https://www.shrm. org/resourcesandtools/hr-topics/talent-acquisition/pages/will-airemove-hiring-bias-hr-technology.aspx.

Lin, Y.-T., Hung, T.-W., \& Huang, L.T.-L. (2020). Engineering equity: How AI can help reduce the harm of implicit bias. Philosophy \& Technology. https://doi.org/10.1007/s13347-020-00406-7
Mann, G., \& O’Neil, C. (2016). Hiring algorithms are not neutral. Harvard Business Review, December 9.

Martin, K. (2016). Understanding privacy online: Development of a social contract approach to privacy. Journal of Business Ethics, 137, 551-569. https://doi.org/10.1007/s10551-015-2565-9

Martin, K. (2018). Ethical implications and accountability of algorithms. Journal of Business Ethics, 160, 835-850. https://doi org/10.1007/s10551-018-3921-3

Mittelstadt, B. (2019). Principles alone cannot guarantee ethical AI. Nature Machine Intelligence, 1, 501-507. https://doi.org/10. 1038/s42256-019-0114-4

Mittelstadt, B. D., Allo, P., Taddeo, M., Wachter, S., \& Floridi, L. (2016). The ethics of algorithms: Mapping the debate. Big Data \& Society, 3, 205395171667967. https://doi.org/10.1177/20539 51716679679

Mujtaba, D. F., \& Mahapatra, N. R. (2019). Ethical considerations in AI-based recruitment. In M. Cunningham \& P. Cunningham (Eds.), IEEE International Symposium on Technology in Society, Medford, MA, USA, November 15-16 (pp. 1-7). IEEE. https:// doi.org/10.1109/ISTAS48451.2019.8937920.

Newman, D. T., Fast, N. J., \& Harmon, D. J. (2020). When eliminating bias isn't fair: Algorithmic reductionism and procedural justice in human resource decisions. Organizational Behavior and Human Decision Processes, 160, 149-167. https://doi.org/ 10.1016/j.obhdp.2020.03.008

Nissenbaum, H. (2004). Privacy as contextual integrity. Washington Law Review, 79(1), 119-158.

Nissenbaum, H. (2009). Privacy in context: Technology, policy, and the integrity of social life. Stanford University.

Noblit, G. W., \& Hare, R. D. (1988). Meta-ethnography: Synthesizing qualitative studies. Sage.

North-Samardzic, A. (2019). Biometric technology and ethics: Beyond security applications. Journal of Business Ethics. https://doi.org/ 10.1007/s10551-019-04143-6

Oswald, F. L., Behrend, T. S., Putka, D. J., \& Sinar, E. (2020). Big data in industrial-organizational psychology and human resource management: Forward progress for organizational research and practice. Annual Review of Organizational Psychology and Organizational Behavior, 7, 505-533. https://doi.org/10.1146/ annurev-orgpsych-032117-104553

Ozkazanc-Pan, B. (2019). Diversity and future of work: Inequality abound or opportunities for all? Management Decision. https:// doi.org/10.1108/MD-02-2019-0244

Park, Y. J. (2013). Digital literacy and privacy behavior online. Communication Research, 40, 215-236. https://doi.org/10.1177/ 0093650211418338

Pena, A., Serna, I., Morales, A., \& Fierrez, J. (2020). Bias in multimodal AI: Testbed for fair automatic recruitment. In 2020 IEEE/ CVF Conference on Computer Vision and Pattern Recognition Workshops (CVPRW), Seattle, WA, USA, June 14-19 (pp. 129137). IEEE. https://doi.org/10.1109/CVPRW50498.2020.00022.

Persson, A. (2016). Implicit bias in predictive data profiling within recruitments. In A. Lehmann, D. Whitehouse, S. Fischer-Hübner, L. Fritsch, \& C. Raab (Eds.), Privacy and identity management. Facing up to next steps (Vol. 498, pp. 212-230). Cham: Springer.

Ployhart, R. E., Weekley, J. A., Holtz, B. C., \& Kemp, C. (2003). Webbased and paper-and-pencil testing of applicants in a proctored setting: Are personality, biodata, and situational judgement tests comparable? Personnel Psychology, 56, 733-752.

Polli, F. (2019). Using AI to eliminate bias from hiring. Harvard Business Review, October 29.

Polli, F., Dolphin, J., \& Kassir, S. (2019). On the basis of brains: How neuroscience and AI advance ethical hiring. Workforce Solutions Review, 10(4), 13-15.

Rąb-Kettler, K., \& Lehnervp, B. (2019). Recruitment in the times of machine learning. Management Systems in 
Production Engineering, 27, 105-109. https://doi.org/10.1515/ mspe-2019-0018

Raghavan, M., Barocas, S., Kleinberg, J., \& Levy, K. (2020). Mitigating bias in algorithmic hiring: Evaluating claims and practices. In Conference on fairness, accountability, and transparency, Barcelona, Spain, January 27-30. New York: Association for Computing Machinery.

Raji, I. D., \& Buolamwini, J. (2019). Actionable auditing. In V. Conitzer, G. Hadfield, \& S. Vallor (Eds.), AAAI/ACM conference on AI, ethics, and society, Honolulu, HI, USA, January 27-28 (pp. 429-435). New York: ACM. https://doi.org/10.1145/ 3306618.3314244.

Recruitment \& Employment Confederation. (2020). Indiscriminate robots. Recruiter - News \& Business Intelligence for Recruiters, 17(04), 2020.

Rhue, L. (2018). Racial influence on automated perceptions of emotions. Social Science Research Network. SSRN 3281765.

Ryan, A. M., \& Derous, E. (2019). The unrealized potential of technology in selection assessment. Journal of Work and Organizational Psychology, 35(2), 85-92.

Sánchez-Monedero, J., Dencik, L., \& Edwards, L. (2020). What does it mean to 'solve' the problem of discrimination in hiring? Social, technical and legal perspectives from the UK on automated hiring systems. In Conference on Fairness, Accountability, and Transparency, Barcelona, Spain, January 27-30 (pp. 458-468). New York: Association for Computing Machinery. https://doi. org/10.1145/3351095.3372849.

Savage, D. D., \& Bales, R. (2017). Video games in job interviews: Using algorithms to minimize discrimination and unconscious bias. ABA Journal of Labor \& Employment Law, 32, 34.

Schumann, C., Foster, J. S., Mattei, N., \& Dickerson, J. P. (2020). We need fairness and explainability in algorithmic hiring. In B. An, N. Yorke-Smith, A. El Fallah Seghrouchni, \& G. Sukthankar (Eds.), 19th international conference on autonomous agents and multiagent systems (AAMAS 2020), Auckland, New Zealand, May 9-13.

Seele, P., Dierksmeier, C., Hofstetter, R., \& Schultz, M. D. (2019). Mapping the ethicality of algorithmic pricing: A review of dynamic and personalized pricing. Journal of Business Ethics. https://doi.org/10.1007/s10551-019-04371-w

Simbeck, K. (2019). HR analytics and ethics. IBM Journal of Research and Development, 63(4/5), 1-12.

Somers, M. J. (2001). Ethical codes of conduct and organizational context: A study of the relationship between codes of conduct, employee behavior and organizational values. Journal of Business Ethics, 30, 185-195.

Suddaby, R., Bitektine, A., \& Haack, P. (2017). Legitimacy. Academy of Management Annals, 11, 451-478. https://doi.org/10.5465/ annals.2015.0101

Suen, H.-Y., Chen, M.Y.-C., \& Lu, S.-H. (2019). Does the use of synchrony and artificial intelligence in video interviews affect interview ratings and applicant attitudes? Computers in Human Behavior, 98, 93-101. https://doi.org/10.1016/j.chb.2019.04.012

Tambe, P., Cappelli, P., \& Yakubovich, V. (2019). Artificial intelligence in human resources management: Challenges and a path forward. California Management Review, 61, 15-42. https://doi.org/10. 1177/0008125619867910

Thielsch, M. T., Träumer, L., \& Pytlik, L. (2012). E-recruiting and fairness: The applicant's point of view. Information Technology and Management, 13, 59-67. https://doi.org/10.1007/ s10799-012-0117-x
Tolmeijer, S., Kneer, M., Sarasua, C., Christen, M., \& Bernstein, A. (2020). Implementations in machine ethics: A survey. ACM Computing Surveys, 53, Article 132. https://doi.org/10.1145/3419633.

University of Montreal. (2018). Montreal declaration for a responsible development of artificial intelligence. https://www.montrealde claration-responsibleai.com/the-declaration.

van den Broek, E., Sergeeva, A., \& Huysman, M. (2019). Hiring algorithms: An ethnography of fairness in practice. In 40th international conference on information systems, Munich, Germany, December 15-18 (Vol. 6).

van Esch, P., \& Black, J. S. (2019). Factors that influence new generation candidates to engage with and complete digital, AI-enabled recruiting. Business Horizons, 62, 729-739. https://doi.org/10. 1016/j.bushor.2019.07.004

van Iddekinge, C. H., Lanivich, S. E., Roth, P. L., \& Junco, E. (2016). Social media for selection? Validity and adverse impact potential of a Facebook-based assessment. Journal of Management, 42, 1811-1835. https://doi.org/10.1177/01492 06313515524

Vasconcelos, M., Cardonha, C., \& Gonçalves, B. (2018). Modeling epistemological principles for bias mitigation in AI systems: An illustration in hiring decisions. In J. Furman, G. Marchant, H. Price, \& F. Rossi (Eds.), AAAI/ACM Conference on AI, Ethics, and Society, New Orleans, LA, USA, February 2-3 (pp. 323-329). New York: ACM. https://doi.org/10.1145/3278721. 3278751 .

Venkatesh, V., Morris, M. G., Davis, G. B., \& Davis, F. D. (2003). User acceptance of information technology: Toward a unified view. MIS Quarterly, 27(3), 425-478.

Wiechmann, D., \& Ryan, A. M. (2003). Reactions to computerized testing in selection contexts. International Journal of Selection and Assessment, 11, 215-229.

Wiggers, K. (2020, March 13). AI weekly: Coronavirus spurs adoption of AI-powered candidate recruitment and screening tools. VentureBeat. Retrieved July 20, 2020 from https://venturebeat.com/ 2020/03/13/ai-weekly-coronavirus-spurs-adoption-of-ai-power ed-candidate-recruitment-and-screening-tools/.

Williams, B., Brooks, C. F., \& Shmargad, Y. (2018). How algorithms discriminate based on data they lack: Challenges, solutions, and policy implications. Journal of Information Policy, 8, 78. https:// doi.org/10.5325/jinfopoli.8.2018.0078

Woods, S. A., Ahmed, S., Nikolaou, I., Costa, A. C., \& Anderson, N. R. (2020). Personnel selection in the digital age: A review of validity and applicant reactions, and future research challenges. European Journal of Work and Organizational Psychology, 29, 64-77. https://doi.org/10.1080/1359432X.2019.1681401

Yarger, L., Cobb Payton, F., \& Neupane, B. (2020). Algorithmic equity in the hiring of underrepresented IT job candidates. Online Information Review, 44, 383-395. https://doi.org/10.1108/ OIR-10-2018-0334

Zafar, M. B., Valera, I., Gomez Rodriguez, M., \& Gummadi, K. P. (2017). Fairness beyond disparate treatment $\&$ disparate impact: Learning classification without disparate mistreatment. In R. Barrett, R. Cummings, E. Agichtein, \& E. Gabrilovich (Eds.), 26th International World Wide Web Conference, Perth, Australia, April 3-7 (pp. 1171-1180). Geneva, Switzerland: International World Wide Web Conferences Steering Committee. https://doi. org/10.1145/3038912.3052660.

Publisher's Note Springer Nature remains neutral with regard to jurisdictional claims in published maps and institutional affiliations. 